Portland State University

PDXScholar

1985

\title{
Lampen und Laternen als Zeichen der Hoffnung in ausgewählten Werken Wolfgang Borcherts
}

Carol Nolan

Portland State University

Follow this and additional works at: https://pdxscholar.library.pdx.edu/open_access_etds

Part of the German Literature Commons

Let us know how access to this document benefits you.

Recommended Citation

Nolan, Carol, "Lampen und Laternen als Zeichen der Hoffnung in ausgewählten Werken Wolfgang Borcherts" (1985). Dissertations and Theses. Paper 3548.

https://doi.org/10.15760/etd.5432

This Thesis is brought to you for free and open access. It has been accepted for inclusion in Dissertations and Theses by an authorized administrator of PDXScholar. Please contact us if we can make this document more accessible: pdxscholar@pdx.edu. 
AN ABSTRACT OF THE THESIS OF Carol Nolan, S.P. for the Master of Arts in German presented August 8, 1985.

Title: Lampen und Laternen als Zeichen der Hoffnung in ausgewaehlten Werken Wolfgang Borcherts.

APPROVED BY MEMBERS OF THE THESIS COMMITTEE:

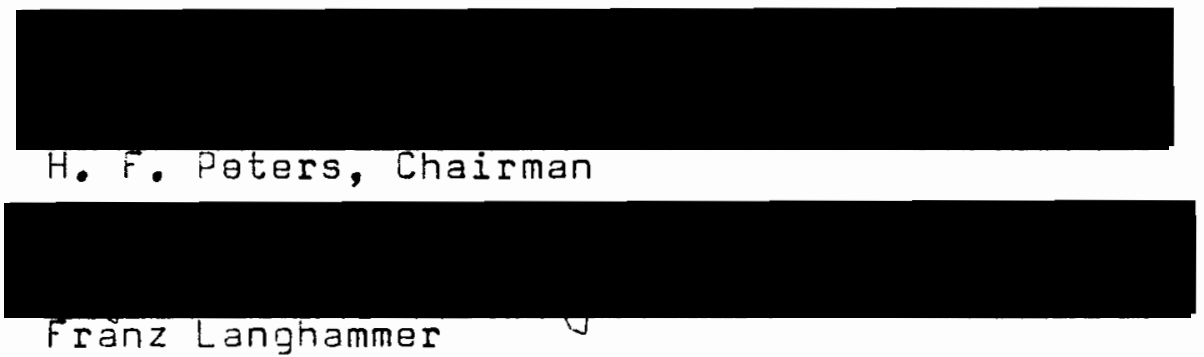

Franz Langhammer

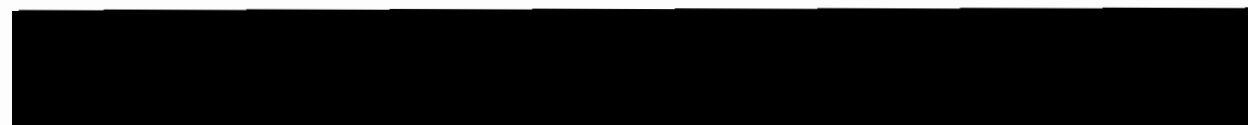

waltetpjogdermann/7d?

.

KIaus Hanson

Wolfgang Borchert wurde 1921 in Hamburg geboren.

Ein vielversprechender Dichter, Schriftsteller und Schauspieler, musste or im zweiten Weltkrieg kaempfen. Er wurde zweimal wegen seiner Antikriegsaussprueche verhaftet, und zwei Jahre nach Ende des Krieges starb er, mit 26 Jahren, an einer sich im Laufe des Krieges zugezogenen Gelbsucht. 
Die Hauptwerke Borcherts bestahen aus Gedichten, Kurzgeschichten und anderen Prosastuecken, und einem Theaterstueck. In diesen Werken sind Zaertlichkeit und Humor, ebenso wie Bittarkeit und Mahnung zu finden.

In den Werken Wolfgang Borcherts werden Lampen und Laternen oft als Zeichen der Hoffnung verwendet. In dieser These wurde versucht, den Kontext und die Bedeutungen dieser Zeichen zu erklaeren. Fuenf Gedichte, acht Prosastuecke und das Theaterstuacke Draussen vor der Tuer wurden untersucht.

In den Gedichten erscheinen die Laternen als Wegweiser in der Dunkelheit, als Quellen der Sicherheit und des Trostes. Man erfaehrt den Wuensch des Dichters, so ein Erleuchteter zu werden. Der Dichter ist sich aber seiner Schwaeche bewusst.

In den Prosastuecken, die im allgemein pessimistischer als die Gedichte sind, erscheinen zwei Gruppen von Menschen: die "Versorgten" und die "Aussenstehenden." Die Versorgten (die Durchschnittmenschen) haben gewoehnlich eine Wohnung und vermutlich einen Lebenszweck. Die Lampen, die ihnen gehoeren, bedeuten also ein Mass von Sicherhait, Liebe und Wohlsein.

Fast jedes Stueck ist aber vom Gesichtspunkt der Aussenstehenden geschrieben. Diese Menschen hoffen auch 
auf die Lampen, obwohl sie wegen ihrer vorhergehenden Erlebnisse oft davon ausgeschlossen sind. Fuer diese gibt es die Laternen.

Diese Aussenseiter in Borcherts Werken geben in schwierigen Situationen nicht auf, sondern gehen immer weiter auf den Strassen des Lebens. Die Laternen sind fuer sie eine Hoffnung auf Liebe und Geborgenheit, aber vor allem bedeuten sie Borcherts eigene Hoffnung auf ainen dauernden Frieden, und eine bessere Zukunft fuer die Menschheit. 
LAMPEN UND LATERNEN ALS ZEICHEN DER HOFFNUNG

IN AUSGEWÄHLTEN WERKEN WOLFGANG BORCHERTS

by

CAROL NOLAN, S.P.

A thesis submitted in partial fulfillment of the requirements for the degree of

\author{
MASTER OF ARTS \\ in \\ GERMAN
}

Portland State University

1985 
TO THE OFFICE OF GRADUATE STUDIES AND RESEARCH:

The members of the Committee approve the thesis of Carol Nolan, S.P. presented August 8, 1985.

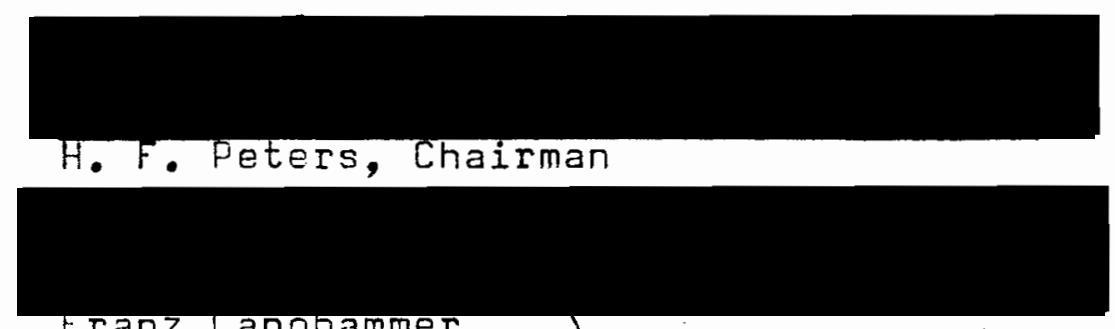

F ranz Langiammer

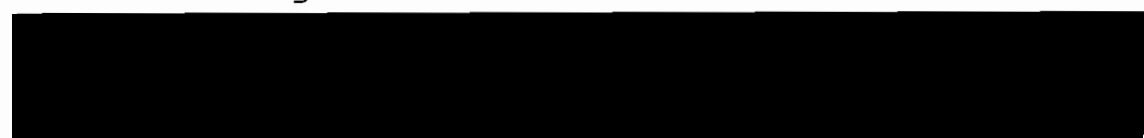

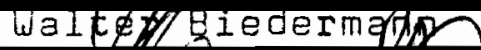

KIaus Hanson

APPROUED:

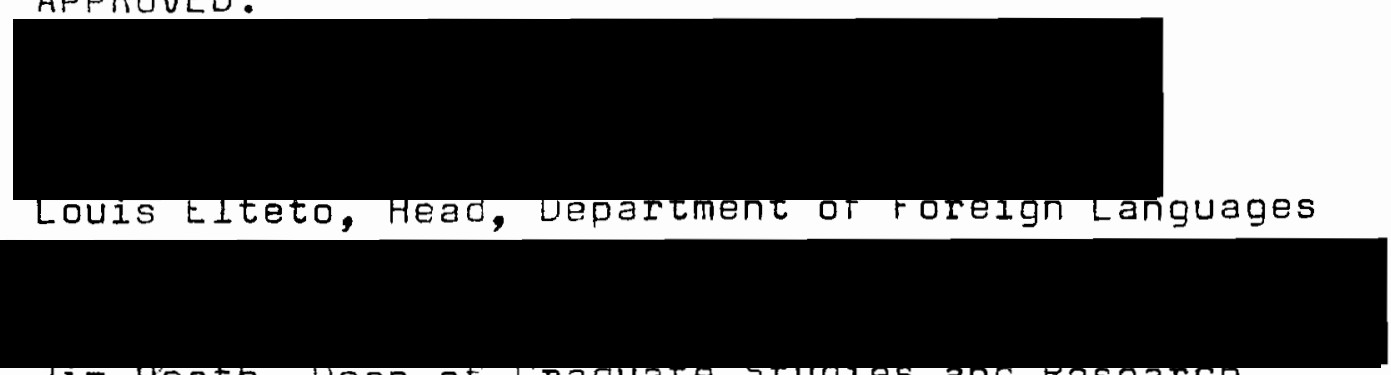

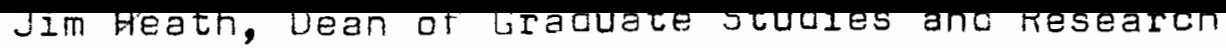


INHALT

SEITE

VORWORT . . . . . . . . . . . . . . . . . . . . . iv EINFÜHRUNG . . . . . . . . . . . . . . . . . . v KAPITEL

I DAS LEBEN WOLFGANG BORCHERTS: "WIR HABEN ES

UNS MIT WOLFGANG BORCHERT ZU EINFACH GEMACHT" 1

II BORCHERTS LITERARISCHER STIL: EINFLÜSSE ANDERER DICHTER . . . . . . . . . . . . . . 11

II LAMPEN UND LATERNEN ALS ZEICHEN DER HOFFNUNG IN DEN WERKEN WOLFGANG BORCHERTS • • 16

Laternen und die Hoffnung in Borcherts

Leben . . . . . . . . . . 16

Die Gedichte . . . . . . . . . 19

Die Prosastücke . . . . . . . . 27

Draussen vor der Tür . . . . . . . 55

IV SCHLUSSFOLGERUNGEN . . . . . . . . . . 68 ANMERKUNGEN . . . . . . . . . . . . . . . . . 73 QUELLEN . . . . . . . . . . . . . . . . . 82 APPENDIX A . . . . . . . . . . . . . . . . . 88 APPENDIX B 


\section{VORWORT}

Die Verfasserin möchte den Mitgliedern der Prüfungskommission, vornehmlich Dr. H.F. Peters, für ihr Interesse danken.

Herzlich zu danken sind auch Frau Irmgard Schindler und Herr Carl-Heinz Corswandt von Hamburg, und Prof. Dr. Alfred Läpple von Salzburg für ihre Beitrăge über das Leben Wolfgang Borcherts.

Die Verfasserin dankt schliesslich der Bibliothek der Universität Indiana für die grosszügige Erlaubnis, ihren reichlichen Bestand zu benutzen, und auch Dr. Elmer Magnuson von der Bibliothek der Portland Staat Universität und Dr. Burmeister von der Staats- und Universitätsbibliothek Hamburg für gütige Hilfe. 


\section{EINFÜHRUNG}

Wolfgang Borchert wurde 1921 in Hamburg geboren. Ein vielversprechender Dichter, Schriftsteller und Schauspieler, musste er im zweiten Weltkrieg kämpfen. Er wurde zweimal wegen seiner Antikriegsaussprüche verhaftet, und zwei Jahre nach Ende des Krieges starb er, mit 26 Jahren, an einer sich im Laufe des Krieges zugezogenen Gelbsucht.

Die Hauptwerke Borcherts bestehen aus Gedichten, Kurzgeschichten und anderen Prosastücken, und einem Theaterstück. In diesen Werken sind Zärtlichkeit und Humor, ebenso wie Bitterkeit und Mahnung zu finden.

In den Werken Wolfgang Borcherts werden Lampen und Laternen oft als Zeichen der Hoffnung verwendet. In dieser These versuche ich, den Kontext und die Bedeutungen dieser Zeichen zu erklären.

Im Laufe meiner Nachforschungen habe ich das Glück gehabt, einen Monat in Hamburg zu verbringen. Während dieser Zeit habe ich die Universitätsbibliothek Hamburg besucht, und auch einen Vetter von Borchert und eine gute Freundin von Borcherts Mutter kennengelernt. Ebenfalls habe ich verschiedene Plätze in Hamburg und Berlin besucht, 
die Beziehungen zu Borcherts Leben haben. Die Beschreibung dieser Erlebnisse ist selber eine Geschichte, und ist auf Seite 91 (Appendix B) zu finden. 
KAPITEL I

DAS LEBEN WOLFGANG BORCHERTS: "WIR HABEN ES UNS MIT WOLFGANG BORCHERT ZU EINFACH GEMACHT." 1

Der Dichter Wolfgang Borchert wurde am 20. Mai 1921 in Hamburg als einziges Kind seiner Eltern geboren. Sein Vater Fritz Borchert war Volksschullehrer als er die sechzehnjährige Hertha Salchow heiratete. Ein lebenslanger Freund von Wolfgang Borchert war sein Vetter Carl-Heinz Corswandt, mit dem ich mich am 28. März 1985, in Hamburg eine Stunde unterhalten habe. Nach Carl-Heinz war Fritz Borchert das Steuerruder der Familie. Von diesem ruhigen und sensiblen Mann lernte Hertha Borchert die Kunst des Schriftstellers, und nach einigen Jahren wurde sie als Heimatschriftstellerin bekannt. Im Gegensatz zu ihrem Mann war Frau Borchert eine sehr offene Persönlichkeit; nach Carl-Heinz Corswandt wurde sie "von der Stimmung abhängig." Wolfgangs Persönlichkeit war der seiner Mutter ähnlich: gewöhnlich sagte er, was er meine, ohne an die Folgen zu denken, und seine Stimmungen waren auch wankelmütig. Seine Eltern gaben ihm keine strenge Erziehung; er durfte an vielen Entscheidungen teilnehmen und wurde als Erwachsener 
behandelt.

Koepke beschreibt Borchert als "an incurable individualist." 2 Wie viele begabte Menschen war Wolfgang Borchert in der Schule kein guter Student. Sein Vetter Carl-Heinz sagt, er hätte es immer schwer gehabt, eine enge menschliche Beziehung zu erlangen, und wäre nicht gerne mit vielen Menschen zusammen gewesen.

Mit 15 Jahren fing Borchert schon an, Gedichte zu schreiben; er ahmte Rilke und später auch Trakl und Benn enthusiastisch nach. Im Jahre 1938 verliess er die Oberschule. Bis 1940 arbeitete er bei der Buchhandlung Boysen in Hamburg. Diese Stellung hat Wolfgang gut gefallen, weil er viele von der Regierung verbotene Bücher lesen konnte. Er wollte aber eigentlich Schauspieler werden, also studierte er während dieser Zeit, "ohne Wissen der Eltern," ${ }^{3}$ an der Schauspielschule Helmut Gmelins. Schon in dieser Zeit wurde Borchert von der Gestapo beobachtet. Im April 1940 musste er eine Nacht auf der Polizeiwache verbringen (er wusste nicht warum), und einige von seinen Briefen wurden von der Gestapo geöffnet. ${ }^{4}$

Ende 1940 bestand Borchert die Schauspielprüfung, und bekam eine Stellung an der Landesbühne Osthannover in Lüneburg, wo meistens Lustspiele aufgeführt wurden. Später nannte er diese Zeit "die schönste . . . meines Lebens." 5

Im Mai 1941 wurde Borchert zum Heeresdienst einberufen, aber in der Armee schrieb er weiter Gedichte. 
Er sagte später, er hätte in seinem Leben "ein paar tausend" geschrieben. 6 Alle seine veröffentlichten Gedichte wurden während der Kriegsjahre geschrieben.

Während seiner Ausbildungszeit in Weimar-Lützendorf wurde er wegen seiner kritischen Briefe nach Hause der Gestapo wiederum verdächtig, aber ehe er verhaftet wurde, schickte man ihn mit seinen Kameraden nach Russland. Dort, in der Nähe von Klin-Kalinin, fanden sie sich, in Temperaturen zwischen 30 und 50 Grad Kälte, ärmlich ausgerüstet und gekleidet. Während dieser Zeit (im Winter 1941-42) kam Borchert wegen einer Schussverletzung der Hand in ein Lazarett. Da der Verdacht bestand, er hätte sich diese Verletzung selbst zugefügt, wurde er im Mai, obwohl er sich kaum von einer Diphtherie erholt hatte, in Nürnberg verhaftet. Nach drei Monatenin einer Einzelzelle machte man ihm den Prozess, dessen Ergebnis entweder Todesstrafe oder Freispruch sein würde. ${ }^{7}$ Er wurde von der Anklage freigesprochen, aber trotzdem musste er wegen der Briefe, die er während der Ausbildungszeit geschrieben hatte, noch sechs Wochen im Gefängnis bleiben.

Im Oktober 1942 wurde Borchert nach Saalfeld und später nach Jena geschickt. In der Nähe von Toropez, in Russland nicht weit von Moskau musste er noch einmal kämpfen. Er beschrieb seine Lage: ". . [D]ie helle Angst hat mich doch oft gepackt, . . denn ich war immer nur auf meine arme harmlose Leuchtpistole angewiesen - also 
praktisch ganz wehrlos." 8 Ende Dezember 1942 wurde er wieder ins Lazarett gebracht. Diesmal hatte er ausser erfrorenen Füssen auch ein eigenartiges Fieber, also fand er sich bald im Seuchenlazarett Smolensk. Während dieser Zeit hat er sich ab und $z u$ besser gefüh1t, und hat auch die Bekanntschaft einiger Mädchen gemacht. "Er berichtete von einer kleinen Russin, Fina, mit der er die Kathedrale von Smolensk besucht hat" und auch von der Krankenschwester Elisabeth, "einer munteren Rheinländerin." 9

Aber er war immer noch sehr krank. Im März 1943 schickte man ihn nach Deutschland zurück: Während dieser Reise musste er stündich Galle erbrechen. ${ }^{10}$ Er blieb im Reservelazarett Elend (Harz) bis August 1943; dann bekam er Urlaubserlaubnis und fuhr nach Hamburg. Erschrocken fand er seine Stadt in Ruinen: kurz vorher war sie durch Bombenangriffe zerstört worden.

Im Oktober, das Gesicht schon "von Gelbsucht verfärbt,"11 war er wieder in Jena. Aber hier kamen die Fieberanfälle so oft und die Leberkrankheit war so stark, dass man beschloss, ihn aus der Armee zu entlassen. (Am Ende seines Lebens war die Diagnose des Arztes, dass Borchert mit einer empfindlichen Leber geboren wurde, und dass diese Empfindlichkeit durch Jahre unzureichender Ernährung und onbehandelter Krankheiten unheilbar wurde.) $)^{12}$ Aber am Vorabend seiner Entlassungstages (30. November 1943) parodierte er auf einem Bierabend den Reichsminister 
Goebbels und wurde von einem Kameraden verraten. Dafür musste er neun Monate im Gefängnis in Berlin-Moabit bleiben. Anfang 1945 hätte Borcherts Kompanie in Hessen kämpfen sollen, aber die offiziere ergaben sich den Franzosen. 13 Borchert entkam mit ein paar Kameraden, und ging, fiebernd und schwach, zu Fuss nach Hamburg.

Dort haben ihn die Eltern mit grosse Freude umarmt. Wieder $z u$ Hause, versuchte Borchert seine Karriere als Schauspieler fortzusetzen. Mit ein paar Bekannten gründete er ein kleines Theater, aber wegen Krankheit und Schmerzen musste er dieses Projekt aufgeben. Im Winter 1945-46 ging er ins Krankenhaus, und obwohl er wieder zurück nach Hause kam, musste er im Bett bleiben.

Da er nicht spielen konnte, fing er an zu schreiben. Während der Jahre 1940-45, im Krieg, in Kälte und Gefangenschaft, hat er immer Gedichte schreiben können. Aber jetzt konnte er keine Gedichte schreiben. Sein Vetter Carl-Heinz meint, er konnte den Mut dafür nicht fassen, da er sich ausgeschlossen von der Welt fühlte.

Dann schrieb er am 24. Januar 1946 "eine von Grund auf eigentümliche, auf Anhieb moderne, ohne jeden Umschweif und ohne Nachkorrektur meisterliche Erzählung: 'Die Hundeblume; "'14 diese wurde im April 1946 publiziert. Durch "Die Hundeblume" wurde Borchert bekannt, und er fuhr fort, weiter zu schreiben. Mileck erklärt, er schrieb "as though possessed."15 Im Laufe des Jahres 1946 schrieb er 
fast dreissig Prosastïke. Unter diesen findet man "Gespräch über den Dächern," "Hinter den Fenstern ist Weihnachten," "Stimmen sind da - in der Luft - in der Nacht," "Die drei dunklen Könige," "Die Stadt," und "Vier Soldaten."16

Unter seinen Bekannten in dieser Zeit is Bernhard Meyer-Marwitz besonders zu erwähnen. Durch seinen Vorschlag wurde "Die Hundeblume" publiziert. Er war auch für die Veröffentlichung der ersten Sammlung von Borcherts im Laufe des Krieges geschriebenen Gedichten verantwortlich. Die Sammlung erschien im Sommer 1946. Unter diesen Gedichten findet man "Ich möchte Leuchtturm sein" und "Laternentraum."

Im Januar 1947 schrieb Borchert sein einziges Theaterstück, Draussen vor der Tür. Ursprünglich ein Hörspiel, wurde es im Februar in Hamburg gesendet. Leider konnte Borchert sein Stück wegen eines Stromversagens in dem Stadteil, wo er wohnte, nicht hören.

\section{Draussen vor der Tür fand nach Rühmkorf eine} "unerwartete Resonanz."17 Nach dieser Sendung bekam Borchert zahlreiche Briefe und Besucher. Er fuhr fort weiter zu schreiben; vom Januar bis September, 1947, schrieb er mindestens 20 Prosastücke: darunter "Die Nachtigall singt" und "Im Mai, im Mai schrie der Kuckuck."18 Die gesamte Liste von Borcherts Prosawerken ist im Appendix $A$ auf Seite 88 zu finden. 
Im Laufe dieser zwei Jahre nach dem Krieg wurde die Lage des schon todkranken Borcherts allmählich schlechter. Er schrieb: "Meine Leber ist ein praller Fussball und mein Kopf ein glühender Teekessel. Das übrige zwischen Fussball und Teekessel ist gereizt und geschwollen wie ein Blinddarm."19 In Hamburg konnte man die notwendige Arznei kaum bekommen; das Klima war ungünstig, und im Winter 1946-47 erlebte Deutschland einebesonders schlimme Kälte. Borcherts Freunde versuchten für ihn eine Erholungsreise in die Schweiz zu ermöglichen.

In dieser Zeit durfte jedoch kein Deutscher über die Grenze, und es dauerte eine lange Zeit, ehe Borchert diese Reise machen konnte. Frau Irmgard Schindler, eine enge Freundin von Hertha Borchert, sagte mir, drei schweizerische Verleger mussten ihm eine offizielle Einladung schicken. Im September 1947 reiste er endich von dem kalten Norden in den Süden. Seine Mutter kam mit ihm bis an die Grenze, aber sie musste in Deutschland bleiben. Nach Frau Schindler hätte er nach Liechtenstein reisen sollen. Als er in Basel ankam, war er so schwach, dass man ihn zum nächsten Krankenhaus bringen musste. ${ }^{20}$ Er kam ins Clara-Spital in Basel, wo ihn fast niemand kannte. Er fühlte sich allein und fremd; erst im Oktober besuchten ihn einige Schweizer, die ihm Kleingeld, Schreibpapier und vor allem Berichte von der Welt draussen gaben. Einer dieser Besucher brachte einige Graphiken von Paul Klee mit, 
um das kahle Krankenzimmer angenehmer zu machen. Aber die zuständige Krankenschwester erlaubte es nicht, diese Graphiken an die Wand hängen zu lassen, also mussten sie auf Stühlen stehen bleiben. Ein Priester, der mehrere Schwestern aus dieser Zeit kannte, hat mir darüber berichtet: "Wolfgang Borchert war deshalb ausserordentlich aufgebracht, so dass es zwischen ihm und seiner Stationsschwester kaum noch zu einem persönlichen Gespräch kam." 21 Man kann sich diese Szene leicht vorstellen: schon von der von ihm so geliebten Welt ausgeschlossen, reagierte der Todkrank stark auf die Verbote, die seinen engen Kontakt mit der Welt beschränkten .

Er befreundete sich mit nur einer Schwester: mit der Pförtnerin Schwester Mina Herbel, die täglich den Patienten ihre Post brachte. 1971 schrieb Schwester Mina: "Von seinen Kriegserlebnissen und den mitmenschlichen Beziehungen aus jener Zeit sprach er sehr selten und nur so nebenbei. Aber seine täglichen Erlebnisse, frohe und andere, sprach er bei mir von seinem Herzen weg. Er war schwer, schwer krank, und litt unsäglich. Dass er anspruchsvoll war, darf ich nicht behaupten." 22

Sein letztes Werk "Dann gibt es nur eins!" schrieb Borchert im Oktober im Clara-Spital. Dieses Stück ist keine Erzählung, sondern ein Manifest gegen den Krieg. Heute steht ein Auszug davon auf einer Tafel in Hamburg neben einer "Friedenseiche," die im Gedenken an den 
"glorreichen Frieden von 1871" gepflanzt wurde.

Borchert starb am 20 November 1947, sechsundzwanzig

Jahre alt. Am nächsten Tag fand die erste Bühnenaufführung von Draussen vor der Tür in Hamburg statt. Bald danach wurde sein Name weltbekannt; Draussen vor der Tür wurde in viele Sprachen übersetzt, und Ende November wurde der Gedichtband An diesem Dienstag veröffentlicht.

Heute liegen die irdischen Überreste Wolfgang Borcherts in dem Ohlsdorfer Friedhof in Hamburg. Drei Schulen sind nach ihm Benannt: die Grundschule in Hamburg-Eppendorf, die er als Kind besuchte, eine Realschule in Velbert (NWR) und eine Realschule in Berlin. Es gibt in Hamburg eine Strasse "Borchertring" und in Berlin einen "Borchertweg."

1976 eröffnete die Universitätsbibliothek Hamburg ein "Borchert-Zimmer," wo verschiedene Denkwürdigkeiten und Schriften über Borchert zu finden sind.

Neue Aufführungen von Draussen vor der Tür Einden noch statt: im Winter 1985 wurde das Stück in München gespielt. 1977 schrieb der ungarische Komponist Sandor Balassa eine Oper auf Englisch: The Man Outside. Die Uraufführung der Oper fand in Budapest statt, und 1983 wurde das Werk in Recklinghausen in Deutschland aufgeführt. Im kommenden Jahr soll eine neue Biographie über Borchert von Claus B. Schröder erscheinen; diese Biographie wird vom Kabel Verlag Hamburg herausgegeben werden. Man 
sieht, die Welt beschäftigt sich noch immer mit diesem ungewöhnlichen jungen Mann. 


\section{KAPITEL II}

\section{BORCHERTS LITERARISCHER STIL: EINFLÜSSE ANDERER DICHTER}

Borchert fing jung an zu schreiben. Als er 15 Jahre alt war schrieb er schon Gedichte. Obwohl Rühmkorf ihn "kaum ein Frühtalent" nennt, ${ }^{1}$ war er für Gedichte begeistert. Wahrscheinlich war sein erstes Vorbild und wichtigster Einfluss der Dichter Rainer Maria Rilke (1875-1926). Auch in Borcherts späteren Dichtungen spürt man Rilkes Einfluss in der Verwendung von Alliteration und Assonanz, wie in seinem Gedicht "Laternentraum:"

- . wo die Mädchen lachen

würde ich wachen

an einem schmalen schmutzigen Fleet $[.]^{2}$

oder in einem anderen: "Draussen:"

Nach draussen muss man schauernd fragen[. $]^{3}$

Koepke erklärt: "Er war in Worte, in Sprache verliebt." 4

Man findet oft den Trochäus in seinen Gedichten, 5 und wie Rilke benutzt er Endreim, iambische Trimeter und Tetrameter, enjambment und "the folk-song stanza" (vier Zeilen, gekreuzte Reime). 6 
Der Regen geht als eine alte Frau mit stiller Trauer durch das Land. Ihr Haar ist feucht, ihr Mantel grau, und manchmal hebt sie ihre Hand. ("Regen") 7 Während der Zeit (1938-40), als Borchert bei der Buchhandlung Boysen arbeitete, lernte er auch den deutschen Expressionismus kennen. Im Gebrauch von Farbe ${ }^{8}$ und in der Nebeneinanderstellung ungleichartiger Bilder ${ }^{9}$ imitierte er Benn, Trakl und Lichtenstein.

$$
\begin{aligned}
& \text { ein müder Hauch } \\
& \text { wie von Zerfall } \\
& \text { umzittert ihn. } \\
& \text { ein blauer Wahn } \\
& \text { webt kühlend } \\
& \text { um die Stirn. ("Danach") } 10
\end{aligned}
$$

Aber besonders in Borcherts nach 1945 geschriebenen Prosastücken und Schauspiel sind die meisten Kennzeichen des expressionistischen Stils zu finden. Einige wichtige Eigenschaften dieses Stils, die man in Borcherts Werk bemerkt, sind:

Abstraktion: ein Charakter scheint mehr eine Figur als eine Person zu sein, z.B. in "Vier Soldaten:" "der Zigarettendreher;" "der in der Ecke."11 
eine traumhafte Eigenschaft: ein Fehlen von innerem

Zusammenhang; eine Umgebung von Unwahrscheinlichkeit; ̈̈bertreibung und das Groteske, wie in Draussen vor der Tür und "Die lange lange Strasse lang."

die stets steigende Unabhängigkeit der Metapher: ${ }^{12}$ die Metapher wird weniger beschreibend, sondern sie äussert die Realität selbst, z.B. in "Die Nacht:" Und wieder geht the dunkelblaue Frau, die blasse Schwester der Betrunkenen und Dichter, durch die verstummten, nebeligen Strassen. ${ }^{13}$

Wiederholungstechnik: die (oft abwechselnde) Wiederholung von Wörten und Sätzen, wie in "Die lange lange Strasse lang:"

Lieber Gott, gib mir Suppe. Lieber Gott, gib mir Suppe. Ein Löffelchen nur. Ein Löffelchen nur. Ein Löffelchen nur. ${ }^{14}$

die Verwendung rhetorischer Fragen, z.B. in Draussen vor der Tür: "Mensch, Mensch, was für eine Strasse bist du?" 15

Die Wiederholungstechnik ist in Borcherts Werken besonders $z u$ erwähnen; McCormick spricht von "a series of mountingly sharp little additions, . . which lead in a circular pattern back to non-complexity and to one overpowering image."16 Jacobs bemerkt die "pointillistische" Eigenschaft von Borcherts Prosa. 
Borchert verwendet seinen mosaikischen Stil in noch einem weiteren Sinn: im ersten Stadium gibt er uns oft nur ein Stück Information über die Figur, die Situation, usw. In jedem folgenden Stadium lernen wir noch etwas darüber, bis wir ein klares Bild davon haben. Ein Beispiel dafür findet man in "Hinter den Fenstern ist Weihnachten."18

S. 96 Ein Soldat geht auf der Strasse, weil etwas ihm nicht zu aushalten ist. (erste Zeile) Er folgt einer Frau. (Es ist Weihnachten.) (Die anderen Soldaten singen und weinen im Bunker.)

S. 97 (Die anderen reden von ihren "Weibern.") Er ist klein (nicht zu klein für den Krieg, sondern für die Liebe). Er folgt ihr (momentan) mit Entschlossenheit. S. 98 (Es ist nass und kalt draussen, und auch im Bunker.) Er hat Hunger und hofft, er kann von ihr etwas zu essen kriegen.

Er hat Angst davor, dass sie ihn ablehnen wird. S. $99 \operatorname{Er}$ ist jung.

Wegen der Rede der anderen Soldaten über ihre "Weiber" wird er so erregt, dass er nicht schlafen kann; deswegen sucht er "ein Weib" auf den Strassen. Wir bekommen die Auskunft über den Mann in kleinen Teilen, und wir hören erst am Ende des Stückes, was man eigentlich "nicht aushält." (S. 96, erste Zeile)

Schliesslich muss man wie viele Kritiker sagen, dass 
Borcherts Werk im allgemein effektiver $z u$ hören ist, als zu lesen. Klarmann bemerkt: ". . The musicality and acoustic keenness which marks his prose."19 Weimar erklärt: "The high frequency of alliteration throughout the play [Draussen vor der Tür] is . . more apposite to radio performance than to stage delivery."20

Obwohl die Prosa gewöhnlich pessimistischer ist als die (früheren) Gedichte, ist Borcherts Stärke der lyrische Charakter seiner Werke; der Klang ist immer wichtig. 21 Nach Koepke ist er ein "verstimmter Lyriker,..22 und Bonwit spricht von der ironischen Verwendung ublicher Versmässe ". . to heighten some particularly glaring contradiction."23 McCormick sagt: "Borchert's prose... reveals the same pronounced rhythmic or musical quality common to his lyric poetry." 24 


\section{LAMPEN UND LATERNEN ALS ZEICHEN DER HOFFNUNG} IN DEN WERKEN WOLFGANG BORCHERTS

"Ich möchte Leuchtturm sein. . bin doch selbst ein Schiff in Not!"1 In diesen Worten von Wolfgang Borchert kann man ein Motiv seines Lebens und seiner Werke sehen. Die Hoffnung kann als "Glauben an die Erfüllbarkeit des Gewünschten" (Wahrig) definiert werden. Manchmal wird die Hoffung als Anker symbolisch dargestellt; auch sieht man als ein Zeichen der Hoffnung eine Harfe, worauf nur eine Saite bleibt. Also wenn die Aussichten am schlimmsten sind, wird die Hoffnung am wichtigsten.

LATERNEN UND DIE HOFFNUNG IN BORCHERTS LEBEN

In seinem kurzem Leben musste Borchert viele Schmerzen ertragen, und seine Werke, besonders die Prosawerke, spiegeln dieses Leiden. Man sollte aber nicht annehmen, dass Borchert ein trauriger oder ein hoffnungsloser Mann war. Im Gegenteil, nach dem Zeugnis von seinem Vetter und lebenslangem Freund Carl-Heinz Corswandt war Borchert im Grunde genommen optimistisch. ${ }^{2}$ Rühmkorf sagt auch, viele von Borcherts Besucher, die 
erwartet hatten, "einen verqüalten Pessimisten" zu sehen, waren erstaunt, so eine "gutartige Freundlichkeit" zu finden. ${ }^{3}$ Die Freundin seiner Mutter, Frau Irmgard Schindler, sagte, er war fröhlich und heiter. Auch im Gefängnis war er "immer sicher;" er wurde nur traurig, wenn seine Eltern ihn besuchten, denn sie mussten von so weither kommen, durften aber nur zehn Minuten bleiben. ${ }^{4}$

Mehrere Kritiker erkennen, dass in Borcherts Werken die Hoffnung, wie auch das Leid, gespiegelt wird. Nach Koepke: "He told stories of survival, he tried to convey a message of hope, although he himself was only feeling his way." 5 Grenzmann spricht von Borcherts Hoffnungsfreudigkeit in den Kurzgeschichten, ${ }^{6}$ und Kaszynski schreibt (über "Gespräch über den Dächern") "Trotz aller düsteren Reflexionen, die in dieser Geschichte fallen, hängen die Helden doch sehr an ihrem 'bisschen Leben. "'7

"Fog-enveloped Hamburg . . furnishes the background for most of Borchert's poems... . [T]here is always a lamp to penetrate the darkness." 8 Als ein "fanatischer Hamburger, "9 wie er sich genannt hat, muss Borchert von Lampen und Laternen sehr beeinflusst worden sein. Wie andere im Norden liegende Hafenstädte ist Hamburg oft dunkel und nebelig. Borcherts Hamburg steht nicht mehr, aber heute haben die Laternen in den Strassen und neben den Flüssen, und die Lampen in den Strassenbahnen eine bestimmte Wichtigkeit. In dieser Gegend hört man ein 
Kinderlied: "Laterne, Laterne, Sonne, Mond und Sterne."10 Borcherts Vetter Carl Heinz erzählt, wie er und Wolfgang oft als Kinder Feuer gemacht haben. "Das Licht und auch die Wärme waren wichtig."11 Der Kritiker Bab schreibt: "Borchert. . liebt die Stadt [Hamburg], . . und er liebt die gelbe Nebelluft und vor allem die Laternen, . . diese Laternen, die in so vielen seiner Verse und Skizzen immer wiederkehren." 12

Es ist also nicht überraschend, dass in den Werken Borcherts zahlreiche Bilder von Lampen und Laternen als Zeichen der Hoffnung erscheinen, auch wenn das Stück selbst ganz pessimistisch ist. Nach Fischert: "The symbol of the streetlight suggests the isolation of this speck of light in the vastness of night and sky and yet the comfort which it provides the city dweller. In a rudimentary fashion it points toward the theme of man's lostness and the infinitesimal hope of redemption which prevails in the stories."13 In diesem Kapitel untersuche ich Bilder von Lampen und Laternen in Borcherts Werken, und versuche, die Bedeutung dieser Bilder als Zeichen der Hoffnung $z$ erklären. 


\section{DIE GEDICHTE}

Die 31 Gedichte in Borcherts Gesamtwerk wurden neben vielen anderen Gedichten während der Kriegsjahre 1940-45 geschrieben. Nach Kriegsende wurden diese Gedichte von seinen Freunden ausgewählt und gedruckt. Der erste Zyklus, Laterne, Nacht und Sterne, wurde im Sommer 1946 verlegt. Von diesem Zyklus werden "Ich möchte Leuchtturm sein," das auf dem Titelblatt steht, und auch "Laternentraum" analysiert.

Die zweite Sammlung, Nachgeschlagene Gedichte, wurde auch von seinen Bekannten später zusammengestellt. Von dieser Gruppe werden "Draussen," "Nachts" und "Die Nacht" untersucht.

"Ich möchte Leuchtturm sein"

Ich möchte Leuchtturm sein in Nacht und Wind für Dorsch und Stint, für jedes Boot und ich bin doch selbst ein Schiff in Not!

Dieses kurze Gedicht, das auf dem Titelblatt von Laternen, Nacht und Sterne steht, wurde 1944 an seinen Freund Carl Albert Lange geschickt. ${ }^{14}$ Es besteht aus sechs Zeilen, und der Reimplan ist unregelmässig; die zweite und 
dritte Zeile reimen, wie auch die vierte und sechste. Diese vier Zeilen sind im iambischen Dimeter geschrieben; Zeile eins und fünf reimen nicht und sind in Trimeter.

In diesem Stück hort man: "Ich möchte . . ." Der Dichter würde den Weg für die anderen Zeigen, und ihnen eine Quelle der Sicherheit sein, aber er ist sich seiner eigenen Schwäche bewusst: ". . . ich bin doch selbst ein Schiff in Not!"

In den Vorstellungen von Schiffen und Leuchttürmen ist Borcherts Zuneigung zum Wasser zu bemerken, und man erinnert sich daran, dass Borchert die Flüsse in Hamburg sehr liebte. Nach seinem Vetter Carl-Heinz haben sie als Kinder an den Ufern der Alster gespielt, als sie noch zu jung waren, an die Elbe zu gehen. In "Die Elbe" erklärt Bochert: ". . [D]ann sagen wir: Elbe! Und wir meinen: Leben!"15 Und gegen Ende seines Lebens schrieb er an seinen Bekannten Bernhard Meyer-Marwitz: "Ich will keine Zeile mehr schreiben können, wenn ich nur mal über die Strasse gehen dürfte, . . und an die Elbe gehen."16

\section{"Laternentraum"}

"Laternentraum," das auf S. 22 zu finden ist, besteht aus fünf Strophen, mit sechs oder sieben Zeilen pro Strophe. Gewöhnlich ist das Reimpaar zu finden, aber in der vierten Strophe sieht man einen "a b b a (c c)" Reimplan. In der dritten Strophe und auch in der letzten 
Strophe gibt es eine siebente Zeile. In der dritten Strophe reimt diese Zeile auf die ersten zwei Zeilen. In der letzten Strophe reimt diese siebente Zeile auf keine andere Zeile. Das Skandieren des Stücks ist unregelmässig. In der dritten Strophe (Zeilen 2,3) ist ein Beispiel von enjambment $z u$ finden.

Wie in "Ich möchte Leuchtturm sein" erscheint die Laterne in diesem Gedicht als eine Art von Menschenhilfe: in der Dämmerung vor einer Tür; für die Menschen im Hafen; für ängstliche kinder.

Das Gedicht beginnt: "Wenn ich tot bin;" dieser Satz wird später im Stück wiederholt. Man sollte sich daran erinnern, dass Borchert die melsten seiner Gedichte im Krieg, in Gefangenschaft und Krankheit schrieb; er muss oft an den Tod gedacht haben. Veilleicht meint er mit diesen Worten, er wolle eine Quelle der Hoffnung nach seinem Tod, ebenso wie in seinem Leben sein. Es ist auch möglich, dass dieser Ausdruck eine Art vom Einsicht bezeichnet, dass man leiden muss, um Menschen helfen zu können. 


\section{"Laternentraum"}

Wenn ich tot bin, möchte ich immerhin so eine Laterne sein, und die müsste vor deiner Türe sein und den Eahlen

Abend überstrahlen.

Oder am Hafen, wo die grossen Dampfer schlafen und wo die Mädchen Iachen, würde ich wachen an einem schmalen schmutzigen Fleet und dem zublinzeln, der einsam geht.

In einer engen

Gasse möcht ich hängen als rote Blechiaterne vor einer Taverne und in Gedanken und im Nachtwind schwanken zu ihren Gesängen.

Oder so eine sein, die ein Kind mit grossen Augen ansteckt, wenn es erschreckt entdeckt dass es allein ist und weil der Wind so johlt an den Fensterluken und die Träume draussen spuken. $\mathrm{Ja}$, ich möchte immerhin, wenn ich tot bin, so eine Laterne sein, die nachts ganz allein, wenn alles schläft auf der Welt, sich mit dem Mond unterhalt natürlich per Du. 17 
"Draussen," "Nachts" und "Die Nacht" gehören zu der Sammlung: Nachgelassene Gedichte.

\section{"Draussen"}

Das macht das Fenster, dass wir 'draussen' sagen und weil wir selber drinnen sind.

Nach draussen muss man schauernd fragen,

denn draussen ist der Wind.

Laternen stehn

schon hundert schwarze Nächte -

und abends, bald nach zehn,

wenn mancher schlafen möchte,

graut wohl die Strasse blass

und schweigend aus der Flut

von Seufzern, Stein und Glas.

Nun ist es unser Blut,

das so gewaltig rauscht -

da hält der Wind im Tanz den Schritt,

bleibt manchmal stehn,

als ob er lauscht.

Und die Laternen gehn

noch lange durch die Träume mit. 18

"Draussen" besteht aus drei Strophen, die im Länge,

Reim und Skandierung unregelmässig sind. In "Draussen"

gibt es keine Tür (wie in Draussen vor der Tür) sondern ein Fenster; hinter dem Fenster (d.h. drinnen) sind alle ausser Gefahr, aber "draussen ist der Wind." Aber draussen stehen auch die Laternen, und obwohl der Wind uns erschreckt, begleiten uns die Laternen, die "schon hundert schwarze Nächte" bleiben. 
"Nachts"

Meine Seele ist wie eine Strassenlaterne

Wenn es Nacht wird und die Sterne

aufgehn, beginnt sie zu sein.

Mit zitterndem Schein

tastet sie durchs Dunkel,

verliebt wie die Katzen

auf nächtlichen Dächern, mit grünem Gefunkel

in den Augen. Menschen und Spatzen

schlafen.

Nur die Schiffe schwanken im Hafen.

Hebt der Mond sich über den Rand

von einem Kirchendache,

ist in meinen Augen

knisternd ein Streichholz aufgeflammt,

und ich Zache.

Regen rinnt-

bei mir sind

nur mein Schatten und der Wind.

Und meine Hände haben noch den Duft

von irgendeinem schönen Kind. 19

"Nachts" besteht aus drei Strophen. Die erste ist lang, mit zehn Zeilen, deren eine aber nur ein Wort

enthält. Die anderen zwei Strophen haben fünf Zeilen. Die Skandierung ist sehr unregelmässig, wie auch der Reimplan. In der zweiten Strophe reimen drei Reimpare, aber in der dritten Strophe reimen alle Zeilen mit einander, ausser dem vierten: rinnt, sind, Wind, Duft, Kind.

In "Nachts" spricht der Dichter, wie in "Ich möchte Leuchtturm sein," noch einmal persönlich: "Meine Seele ist 
wie eine Strassenlaterne." Nach der ersten Strophe ist vollständige Personifizierung der Laterne zu bemerken; die Laterne spricht wie eine Person. "Hebt der Mond . . ist in meinen Augen knisternd ein Streichholz aufgeflammt, und ich lache." Auch sind Assonanz und Alliteration zu finden: ". . auf nächtlichen Dächern, mit grünem Gefunkel . . ."

Der Ton dieses Gedichtes ist etwas leichter als der von "Draussen." Es gibt Mond und Sterne (auch Wind), und man fühlt nicht, dass die Nacht gefährlich ist; über die Strassenlaternen in "Nachts" schreibt Fickert: " . . [I]t fulfills its purpose in casting a gleam into the darkness which encompasses human life. Another function of the streetlight is to laugh in affirmation of life."20

"Die Nacht" ist auf S. $26 \mathrm{zu}$ finden, und besteht aus vier dreizeiligen Strophen. Reimplan und Skandierung sind unregelmässig; nur zwei Zeilen reimen miteinander: die zweite und die vierte, die aber nicht in derselben Strophe stehen. In diesem Stück sind Assonanz und Alliteration auch zu finden: "die dunkelblaue Frau;" "es schwankt im Schlendrian . . ." In "Die Nacht" scheint die Nacht nicht als etwas furchterregend, sondern als "die blasse Schwester der Betrunkenen und Dichter." Die Betrunkenen umarmen "verzweifelt und berauscht" die Laternen, um einem Sturz zu entgehen; also sind die Laternen den Betrunkenen wie ein Anker. 
"Die Nacht"

Und wieder geht die dunkelblaue Frau, die Blasse Schwester der Betrunkenen und Dichter, durch die verstummten, nebeligen Strassen.

Es schwankt im Schlendrian das Nachtgelichter:

Die Mädchen, die für Stunden heilig sind, glühn sündhaft aus dem Häuserschatten, bis sie der kühle Morgenwind verseucht. Laternen fühlen sich von den Bezechten verzweifelt und berauscht umarmt der Dichter aber flüstert seinen grossen Monolog: Nimm, dunkelblaue Frau, die ohne Ruhe sind, in deinen gnadenreichen Schoss! 21

Im allgemeinen sieht man in diesen fünf kurzen Gedichten entweder eine literarische Personifizierung der Laterne als eine Quelle der Hoffnung in dem Dunkel, oder den Wunsch des Dichters, so ein Erleuchter zu werden. Wie Klarmann sagt: "Borchert loves the symbol of the lantern to which he returns again and again, and with which he identifies himself." 22 Manchmal ist der Ton des Gedichts ernst; oft aber scheint der Dichter zu lächeln.

Man kann auf ein wichtiges Element in Borcherts Stil noch einmal hinweisen: beim Rezitieren der Dichtungen ist die Unregelămssigkeit der Skandierung völlig zu spüren. Besonders in "Nachts" versteht man die Meinung von Edward Allen McCormick, dass in einigen von Borcherts Gedichten die Poesie in Gefahr ist, sich in Prosa aufzulösen. ${ }^{23}$ 


\section{DIE PROSASTÜCKE}

Nach Carl-Heinz Corswandt schrieb Borchert seine Gedichte hauptsächlich als "Nebenprodukte;"24 er wollte eigentlich Schauspieler werden. Aber nach Kriegsende musste er zugeben, dass er diesen Lebenstraum nicht durchführen konnte. Und plötzlich konnte er keine Gedichte mehr schreiben. In dieser ernsten Zeit befasste er sich mit den unzähligen Erinnerungen, die er besonders während der Kriegsjahre gesammelt hatte, und er fing an, Prosa darüber zu schreiben. Koepke sagt: "Borchert wrote only about the world he knew: prison, the Russian front, Hamburg after the war." 25 Nach Carl-Heinz Corswandt gibt es ein Körnchen von Wirklichkeit in jedem von Borcherts Stücken. Mileck nennt das Prosawerk: ". . [an] earnest appeal to his generation of Germans ...."26 und weiter:

". . [H]e was intent only upon truth: upon portraying the human condition within the framework of war and its aftermath." 27

Viele Kritiker stimmen $z u$, dass Borcherts Prosawerke den Gedichten überlegen sind. Nach McCormick: "By common agreement his lyrics are relatively immature, sometimes imitative, never so forceful or convincing as his prose."28 Ebenso Klarmann: "Borchert is at his lyrical best when he does not write poetry."29 In "In Sachen Wolfgang Borchert" sagt Koepke: ". . [S]einen Gedichten fehlte die Anschaulichkeit. Auch diese Gedichte versuchten alles, das 
ganze Leben auf einmal, auszusprechen, und da ihnen das mislang, wurden sie leer. Jetzt, 1946, von der Hundeblume $a b$, ist diese Anschaulichkeit da, Situationen werden deutlich, Gegenstände sind da.. . "30 In "German Writers after 1945" schreibt Koepke weiter: "Borchert's stories . . demonstrate his astonishing artistry, which was overlooked at first when his works seemed nothing but a slice of life." 31

Nach mindsetens zwei Kritikern ist diese "artistry" das Resultat der Einfachheit von Borcherts Sprache. Fickert schreibt: ". . [T]he words are massive in their simplicity," 32 und McCormick erklärt: ". . [S]eldom do we find a writer who is able to do as much with as limited a stock of themes. . His achievement in this respect is outstanding. . . If and when those themes become exhausted it will surely be Borchert's language, his ability to offer a lexical metaphor for the appalling simplicity and stark essentiality of life, that will grant him a second hearing." 33

In dieser Arbeit werden Draussen vor der Tür und acht Prosastücke analysiert: "Gespräch über den Dächern," "Hinter den Fenstern is Weihnachten," "Stimmen sind da - in der Luft - in der Nacht," "Die drei dunklen Könige," "Die Stadt," "Vier Soldaten," "Die Nachtigall singt," und "Im Mai, im Mai schrie der Kuckuck." Fünf von diesen entstanden im Jahre 1946; die anderen zwei Werke schrieb 
Borchert im Jahre 1947, nach Draussen vor der Tür.

"Gespräch über den Dächern"34

"Gespräch über den Dächern" gehört zu dem 1947

verlegten Band Die Hundeblume, in der Gruppe "Die

Ausgelieferten." "Gespräch" ist ein Dialog, und zwar fast ein Monolog. Zwei Männer unterhalten sich in einem Zimmer in der Stadt, aber der erste spricht erst gegen Ende des Stücks.

Ausserhalb des Zimmers ist es dunkel. Die Laternen sollen die Bürger vor Gefahr schützen:

In den Strassen stehen die Lampen und passen auf, Dass nichts passiert.

Das Zimmer ist auch dunkel:

Im Auge [des zweiten Mannes] blinkt zage das Licht von der Lampe im Hof. Aber der Hof, das ist draussen und die Lampe glimmt sparsam. Der sprechende Mann scheint ohne Hoffnung zu sein. Er sieht, dass das Leben sehr kurz und unsicher ist, obwohl die Menschen leben, als ob es nicht so wäre. Besonders in der Stadt ist der Einzelne "namenlos, zahllos, wahllos," unwichtig. Borchert vergleicht das Stadtleben mit der Dunkelheit der Nacht.

Verlaufen auf dieser Welt, ohne Herkunft, ohne Zuhause. Beschenkt an die antwortlose einsame Nacht in den Strassen. 
Diese elenden Stadtbewohner suchen Liebe, Leben, und Gott, aber sie werden immer enttäuscht.

Wir warten jede Nacht auf die Sonne.

Wir warten bei jeder Lüge auf die Wahrheit.

Die Leute sollten Hilfe, Belehrung bekommen, aber im

Gegenteil irren sie umher, "mondlos, sternlos im Dunkel, mit arm-seligen schwindsüchtigen Laternen betrogen."

Diese Laternen sind also eine Enttäuschung;

veślleicht verweist Borchert hier auf Deutschlands

Führerschaft. Aber während man auf die Sonne der Wahrheit wartet, kann man es bis morgen aushalten:

Und morgen passiert es vielleicht,

morgen kann es schon geschehen.

Ein Schiff bauen, eine Schaufel brauchen. . .

Es ist $z u$ erwähnen, dass später in diesem Stück noch einmal die Laterne erscheint. Der erste Mann schlägt dem anderen vor, er sollte sich aufhăngen, weil er so verzweifelt ist. Er bekommt als Antwort:

Hast du nicht begriffen, nie begriffen, dass ich dieses Leben doch liebe. Mein Gott, und ich und die Laterne!

Wahrscheinlich hatte der erste Mann die Frage gestellt, damit der zweite seine eigene Einstellung erklären könnte. Der erste Mann sitzt, ruhig und schweigend, "tief im Zimmer," im Gegensatz zu dem zweiten, der rastlos am Fenster steht. 
Diese Situation kann man verschieden interpretieren. Einmal kann man in dem ersten Mann einen, der nicht an die Welt, sondern nur an sich selbst denkt, sehen (Diese Art von Mensch erscheint auch in Draussen vor der Tür und "Im Mai, im Mai."); und in dem anderen einen, der sich um die Welt Sorgen macht. Anderseits kann man in dem ersten Mann einen erkennen, der nach Klarmann "silent, warm, secure, sound and at peace with himself and the world" ist, und in dem anderen einen "intellectually corroded by his time, by too much thinking, too much asking, too much concern with life in the abstract . . " 35 Da der erste Mann dem zweiten schliesslich hilft, ist die zweite Deutung vielleicht besser. Dieser Mann wird durch seine Fragen und sein "Dasein" eine Lampe für den zweiten. Am Ende dieses Stücks kommt der Morgen, und die Lampen und Sterne werden unnötig und blass.

In "Gespräch" ist die poetische Eigenschaft von Borcherts Prosa leicht zu finden. Man hört Alliteration und Assonanz:

Und unser Leben, unsere Liebe und unser geliebtes gelebtes Leid - sie sind ungewiss und zufällig wie die Welle und der Wind. Willkürlich.

Und draussen da steht die Stadt. Dumpf, dunkel, drohend. Die Stadt: Gross, grausam, gut. Die Stadt: Stumm stolz, steinern und unsterblich. 
McCormick spricht von Borcherts Übergang von Poesie zu Prosa, und bemerkt in der Prosa "the same pronounced rhythmic or musical quality common to his lyric poetry." 36 In diesem Stück hört man auch diese rhythmische musikalische Prosa:

Und der Zufall, der unberechenbare verspielte Gott über uns, der Zufall, der grausame gewaltige Zufall balanciert betrunken auf den Dächern der Welt.

Viele Merkmale des Expressionismus sind im "Gespräch" zu finden. Man kennt die Namen der zwei Männer nicht. Sie werden als "der am Fenster" und "der im Zimmer" oder als "der Blonde" genannt. Es ist aber nicht anzunehmen, dass sie nur als Figuren stehen. Da dieses Stück Bernhard Meyer-Marwitz gewidmet wurde, ist es möglich, dass die zwei Männer ihn und Borchert darstellen.

Der Mann am Fenster spricht in einem rhetorischen

Stil. Viele Beispiele von Wiederholung sind zu bemerken: Brüllt in der Stille der Nacht, brüllt in der Stille der Liebe, brüllt in der stummen Einsamkeit.

Wir lachen. Und unser Tod ist geplant vom Anfang an. Wir lachen. Und unsere Verwesung ist unausweichlich. Wir lachen. Und unser Untergang steht bevor. Und der Tod, und der Tod, und der Tod lacht. 
Man hört auch die (nicht ganz) rhetorischen Fragen:

Sind wir ohne Antwort? Sind wir, wir selbst, diese Antwort? . . Sind wir uns selbst, uns selbst ausgeliefert?

Diese Fragen werden dem ruhigen Mann gestellt. Er antwortet aber nicht, denn "[d]er Frager am Fenster hat sich mit seiner Frage selbst geantwortet."

"Hinter den Fenstern ist Weihnachten"37

Nach Rühmkorf schrieb Borchert "Hintern den Fenstern ist Weihnachten" im Jahre 1946,38 obwohl dieses Stück im Band "Die traurigen Geranien" und andere Geschichten aus dem Nachlass zu finden ist. In diesem Band erscheint eine andere, sensiblere Seite von Borchert als die, des Dichters des Aufschreis. 39

Im Nachwort dieses Bandes erklärt Rúhmkorf, dass "der Gattungsanspruch dort am ehesten erfüllt scheint, wo eine Kurzgeschichte aus dem Augenblick lebt und wo auf der Schneide des Moments ein Schicksal sich entscheidet."40

"Hinter den Fenstern" spielt in einer nebligen Stadtstrasse. Der Schriftsteller spricht in der ersten Person. Er folgt einem hübschen Mădchen, und er stellt sich vor, dass er mit ihr spricht. Seine Gedênken wechseln oft, und wir erfahren, dass er ein junger Soldat ist; er wohnt in einem Bunker, wo alles "nach Tabak und Leder und nassen Klamotten" riecht, wo "die andern" immer "von ihren 
Weibern" sprechen, und wo sie heute abend Weihnachtslieder singen und weinen. Er ist in die Stadt gegangen.

Er möchte auch ein "Weib" haben, und er will dieses Mädchen danach fragen. Beide sind "ganz allein in der Stadt." Alle anderen sind zu Hause, "hinter den Gardinen," wo es Weihnachten ist: manchmal sieht man ". . die Kerzen vom Tannenbaum." Der Soldat stelit sich vor, wie es sein würde, wenn er mit ihr nach Hause gehen könnte. "Dann sitzen wir nebeneinander auf deinem Bett. Und der Nebel und die Kälte stehen vor der Tür."

"Die Laternen stehen Posten." Bei jeder Laterne kann der Soldat ein bisschen mehr von dem Mädchen sehen: die Augen, die Beine, die Knie. Bei jeder Laterne hofft er wieder; bei jeder Laterne kehrt das Mädchen aber den Kopf weg. Er will sich vorstellen, aber jetzt hat er Angst vor der Ablehnung, weil er klein und jung ist. Die Laternen, die früher etwas waren, worauf er sich freuen konnte, werden jetzt Herausforderungen.

Die "Schneide des Moments" kommt: "Bei der nächsten Laterne rede ich dich an. . . " "Da kommt die Laterne. . . Die Laterne kommt. . Da ist die Laterne. - . Die Laterne." Aber der junge Soldat ist nicht mutig genug, sein Glück zu versuchen, und nach diesem spannenden Aufbau bleibt er unentschlossen: "Nein, ich warte noch ein paar Laternen."

Er spricht noch davon, dass er mit dem Mădchen 
sprechen will. "Wenn die nächste Laterne kommt, . . ." Aber der Höhepunkt des Stücks ist vorbei, und wir wissen nicht, ob er eigentlich etwas sagt oder nicht. In diesem Stück ist also die Laterne eine Quelle der Hoffnung: solange es Laternen gibt hofft der junge Soldat noch, er kann das Mädchen kennenlernen, auch wenn er den Mut nicht hat, diese Hilfe zu benutzen. Auch sind die "Kerzen vom Tannenbaum" nicht zu übersehen: sie sind doch die Weihnachtslampen, und sie stellen eine Hoffnung auf Wärme und Frieden dar. Der Soldat hofft, er kann durch die Laternen eine Tür öffnen und endlich "hinter den Fenstern" sein, wo es Weihnachten ist.

"Stimmen sind da - in der Luft - in der Nacht"41

"Stimmen sind da - in der Luft - in der Nacht" gehört zu der Gruppe "Die Ausgelieferten," die im Jahre 1946 im Band Die Hundeblume entstand. Die Erzählung spielt in einer Strassenbahn an einem Novembernachmittag. Draussen sind neblige Stadtstrassen. In der Strassenbahn sitzen sechs Leute: "fünf [von dem Nebel] Entronnen" und der Schaffner. Sie sitzen da, warm, unter den "tröstlichen trüben Lämpchen," aber sie sind ganz allein und isoliert voneinander.

Unter den Insassen findet man einen älteren Herrn, eine alte Frau, zwei junge Mädchen und einen jungen Mann, der "blass. . . unter dem trüben Lämpchen" aussieht. Er 
scheint zu schlafen. Der ältere herr spricht viel über die Stimmen der Toten in der Nacht. Der Schaffner und die alte Frau stimmen ihm zu. Die zwei Mädchen kichern nur, weil sie an lebendige mănnliche Stimmen in der Nacht denken.

Der junge Mann scheint noch zu schlafen. Dadurch wird der Åltere verärgert; er sagt: "Ja, die Jungen! Die können schlafen. . . Die hören die Toten nicht. . . Nur wir Alten haben inwendig Ohren. . ."

Dann öffnet der junge Mann die Augen und steht auf und nähert sich dem Älteren. (Csuri sagt, die Geschichte erreicht hier den Höhepunkt. ${ }^{42}$ ) Der Altere wird darüber entsetzt, aber der Junge sagt ihm nur: "Oh, bitte. Werfen Sie nicht die Zigarette weg. Geben Sie sie bitte mir. Mir ist schlecht. Ich habe nämlich etwas Hunger."

Der Ältere wird plötzlich mitfühlend. Nach Csuri ersieht er die Unrichtigkeit seines Urteils, "ohne dass 'der junge Mann' auf seine Annahme in direkter Form reagiert hat." 43

"Da feuchten sich die Tränensacke an . . ." Weil der Junge keinen Mantel trägt, fragt der Altere danach. Der Junge antwortet: " . . meine Mutter sagt jeden Morgen zu mir, ich soll den Mantel anziehen.. . Aber sie ist schon drei Jahre tot. Sie weiss ja nicht, dass ich keinen Mantel mehr habe. . . ."

Der Junge nimmt die Zigarette und steigt aus dem Wagen. "Er hatte Hunger. Er hatte keinen Mantel. Seine 
Mutter war tot, und es war November."

In "Stimmen sind da" ist die (beleuchtete) Strassenbahn als ein Zeichen der Hoffnung auf die Sicherheit zu verstehen. Schmidt erklärt: ". . . man könne in ihr geborgen sein."44 cu dieser Lage tragen die "tröstlichen" Lampen eine gewisse Heiterkeit bei; Csuri setzt den Lampen die düstere Umgebung draussen gegenüber. ${ }^{45}$

Auch in dieser Erzählung erleuchtet die Lampe die Gestalten der Figuren: "Sehr blass sass er [der junge Mann] da unter dem trüben Lämpchen." Auch wären die anderen an diesen nebligen Nachmittag ohne die Lampen wahrscheinlich schwer zu sehen.

Aber erst in der beleuchteten Strassenbahn gibt es die Möglichkeit zur Kommunikation. Der ältere Herr spricht über die Stimmen". . . in der Luft. In der Nacht. . . Die Toten sind es."

Alle hören ihn. "Die anderen [ausser dem jungen Mann] . . hielten den Atem an." Die Mädchen kichern; die alte Frau und der Schaffner stimmen ihm $z u$, aber wir wissen nicht, ob sie den $\dddot{A} l t e r e n$ eigentlich gehört haben oder ob sie zustimmen: es könnte doch nur Höflichkeit oder Angst sein. Wir wissen auch nicht, ob sie den Alten überhaupt verstehen.

Als aber der Altere über den Jungen ein schlechtes Urteil fällt ("Ja, die Jungen! Die können schlafen. . . Die hören die Toten nicht. . . "), steht plötzlich der 
Junge auf. Der Ältere fühlt sich bedroht, aber der junge Mann bittet nur um die Zigarette des Alten. Ihm ist vor Hunger schlecht.

Dieser Junge hat vielleicht den alten auch nicht gehört; er hat nicht zugestimmt. Aber wir wissen ohne Zweifel, dass er die Stimmen der Toten doch hört. "Meine Mutter sagt jeden Morgen zu mir, ich soll den Mantel anziehen. . Aber sie ist . . tot." Der junge Mann hört wahrscheinlich kaum die Stimme der Lebenden, sondern nur die der Toten. ${ }^{46}$ Er nimmt das kleine Licht und verlässt allein die Strassenbahn. Nach Csuri ist die Zigarette ein Zeichen des Alleinseins des Jungen: "Seine Hoffnungslosigkeit und Ziellosigkeit 'draussen' . . wird durch die im 'Nebel' allein orientierende 'glimmende Zigarette' als Opposition zu den 'tröstliche(n) trübe(n) Lämpchen' des 'inneren Raums' in negativer Richtung klar angedeutet. 147

Doch ist die Zigarette ein Licht; für den jungen Mann bedeutet sie wenigstens eine Hoffnung auf seine Gesundheit, und er bringt etwas Tröstliches in den Nebel aus der Strassenbahn mit.

In diesem Stück trägt die Alliteration sehr zu der Stimmung bei: ". . [D]ie Strassen waren leer und lärmlos und ohne Lust." "Draussen war Nebel, war Nachmittag und November." 
"Die drei dunklen Könige" 48

Die im Jahre 1946 geschriebene Erzählung "Die drei dunklen Könige" ist auch, wie "Hinter den Fenstern," eine Weihnachtsgeschichte, und gehört zu dem Zyklus An diesem Dienstag in der Gruppe "Im Schnee, im sauberen Schnee." Die meisten dieser Erzählungen handeln von der russischen Front des Krieges. Auch eingeschlossen in dieser Gruppe sind die Prosastücke "Die Stadt" und "Vier Soldaten."

"Die drei dunklen Könige" spielt in einer Vorstadt, deren Gebäude durch den Krieg zerstört sind. Ein Mann, seine Frau und ihr neugeborener Sohn leiden an Kälte und Hunger, und die Eltern haben Angst vor der Zukunft. Der verzweifelte, verärgerte Mann möchte jemanden "die Fäuste ins Gesicht schlagen," aber "er hatte keinen." (Zimmerman erklärt: "Welche Finsternis muss in der Seele eines Mannes herrschen, der immer wieder bedauert, niemanden zu haben, 'dem er dafür die Fäuste ins Gesicht schlagen konnte' !") 49

Der Mann bringt ein Stück Holz nach Hause, und zündet es im Ofen an. Es wirft "eine Handvoll warmes Licht," das auf das Kind fiel. Die Mutter denkt, "er lebt."

Dieses Licht (die einzige "Lampe" im Haus, nimmt man an) wird aber von draussen bemerkt, und bald stehen drei Soldaten an der Tür. "Wir sahen das Licht. . vom Fenster," sagen sie. Kaszynski sagt: "Sie kommen aus der Nacht, . . ziehen aber ans Licht." 50 Sie wollen nur zehn 
Minuten nahe der Wärme bleiben.

$$
\text { "Dann fiel das Licht auf sie." }
$$

Jetzt kann die Familie ihr Leid mit dem der Heimkehrer klar vergleichen. ${ }^{51}$ wie die junge Familie leiden diese Drei an Kälte, Hunger, und Angst: einer hatte keine Hände: "Erfroren, sagte er;" einer hatte geschwollene Füsse: "Wasser . . vom Hunger." "Der dritte zitterte. . das sind nur die Nerven. Man hat eben so viel Angst gehabt."

Die Besucher teilen ihre ärmliche Habe mit ihren Gastgebern: für den Mann eine Zigarette: "dann gingen die vier vor die Tür und ihre Zigaretten waren vier Punkte in der Nacht." (Das Licht schafft plötzlich eine Verbindung unter den Männern: während dieser Zeit sind sie nicht, einer mit Kälte, einer mit Hunger, usw., sondern eine Gemeinschaft.) $)^{52}$ Das Kind bekommt ein Spielzeug aus Holz. "Ein Esel. . ich habe sieben Monate daran geschnitzt." Und "zwei gelbe Bonbons. . . für die Frau." Als das Kind zu schreien anfängt, verlassen die drei Soldaten das Haus. Der Mann bleibt verärgert, "aber er hatte kein Gesicht für seine Fäuste." Aber die Frau ist ermutigt, weil das Kind laut geschrieen hat: "Ich glaube, er lacht." (Man kann das mit "er lebt," S. 186 vergleichen.)

Die drei bleiben zusammen in der "Handvoll Licht." "Heute ist ja auch Weihnachten."

In dieser Erzählung kann man das Licht, die "Lampe," als den Kern der Geschichte und der Hoffnung, die sie 
enthält, verstehen. Dieses Licht erhellt die Persönlichkeit der Erzählung und führt wie der Stern von Bethlehem die Soldaten zu dem Haus. Die Lage der Familie bleibt nach dem Besuch der Soldaten genau wie vorher, und doch hat sich die Stimmung wesentlich geändert. Pichl sagt etwas Ähnliches: "So verwandelt sich in den 'Drei dunklen Könige' der locus terribilis nach dem $\mathcal{S}$ chreien des Kindes im subjektiven Empfinden der Mutter in einen locus amoenus, obwohl sich sie Ausstattung des Raumes nicht ändert." 53

Das Licht erleuchtet auch das Gesicht des Kindes, das als ein Zeichen neuer Hoffnung gesehen werden kann. Ausserdem zitiert Kaszynski Albrecht Weber: "Mit den Geschenken der 'dunklen Könige' werden sich Vater und Mutter des Heiligen dieser Nacht bewusst, des unendlichen Reichtums auch, den sie aneinander und miteinander am Kind haben, unbegriffenen Reichtums . . Begnadet auch gehen die Drei; denn 'sie sahen das Licht,' das in die Finsternis des kreatürlichen Angst leuchtet . . ."54

Über dieses Stück spricht Zimmerman von der Angemessenheit der Sprache. Diese Welt ist eine zerstörte Welt, und ". . die Sprache scheint selbst am meisten gelitten $z u$ haben, sie ist selbst verarmt, erkaltet, zertrümmert, und sie muss versuchen, aus den Trümmern - . ein neues Bild des Menschen und der Welt aufzurichten, . . ein Bild, das den Vorzug besitzt, der Wirklichkeit der Dinge näherzukommen." 55 
Zimmerman weist auch darauf hin, dass die Dinge in dieser Welt personifiziert sind und auch leiden: "Das Pflaster war erschrocken,' 'die Latte seufzte auf,' und '. . sie weinte dabei, die Tür."1156

Nach Migner zeigen Borcherts Dichtungen auch ". . den Versuch, . . alte. . Ordnungen hinter sich zu lassen und zum Bau einer neuen Welt zu gelangen." 57

\section{"Die Stadt"}

Vermutlich im Jahre 1946 geschrieben, wurde "Die Stadt" im Sommer 1947 im Band Die Hundeblume in der Gruppe "Unterwegs" herausgegeben.

In diesem Stück kann man "den [jungen] Nächtlichen" als den Schriftsteller verstehen. Er ist neben den Geleisen "unterwegs." Der Mond gibt Licht; auch sieht er "das Helle hinten am Himmel:" die Stadt.

Der Nächtliche ist allein. Aber dann kommt "der Mann mit der Lampe." Er ist älter: "Na, Junge, wohin denn?" "Hamburg."

Der Altere versteht, die Stadt bedeutet für diesen jungen Mann Helligkeit und Freude. Deswegen warnt er: "Aber unter den hellen Laternen gehn auch nur welche, die Hunger haben."

Der Junge hat nicht viel darüber nachgedacht, aber er antwortet etwas trotzig: "Dann muss man wieder hin!. . . Das ist das Leben!" 
Der mit der Lampe ist pessimistisch; für ihn ist das Leben eine Reihe von alltäglichen Erlebnissen, die schnell vorbeigehen. So ein erfülltes Leben, erwidert er sarkastisch.

Dann sagt der Junge: "Das Leben ist mehr, als im Regen laufen und nach Türdrückern fassen. . . Das Leben ist: Angst haben. Und Freude haben. . . Freude, dass man weitergehen kann."

Die zwei erreichen ein kleines Haus: die Wohnung des Alteren. "Der Mann machte die Lampe kleiner. . 'also, Hamburg!" Der Junge geht weiter nach Hamburg: "ein heller Fleck . . am Himmel."

In "Die Stadt" scheint die Lampe des älteren Mannes als eine nicht ganz notwendige Reisehilfe; der Mond scheint doch hell. Aber diese Lampe, die der ältere Mann bringt und hält, erleuchtet "die beiden Gesichter" und erleichtert die Mitteilung $z$ wischen den zwei Männern.

Unter der "Helle am Himmel" versteht der Junge das freudige Leben der Stadt. Die Laternen in den Strassen von Hamburg spiegeln diese Helle wieder; sie bedeuten Hoffnung für den jungen Mann. Er scheint Hamburg nicht gut zu kennen: "Dann ist das andere gleich," Der ältere Mann kennt die Stadt wahrscheinlich gut. Er trägt seine eigene Lampe, und vielleicht hat er auch Hunger.

Der Ältere kommt nach Hause; er macht die Lampe kleiner, denn er brauchte ihr Licht nur für die Reise. Der 
Junge geht weiter, mit vielleicht einer anderen Vorstellung von der Stadt, als er vor kurzem noch hatte.

In dieser Erzählung erscheinen die Lampen und

Laternen von Hamburg als Hoffnung auf Freude für den jungen Mann. Die Lampe des alten Mannes ist auch eine geistliche Erleuchtungshilfe, eine wichtige Quelle der Hoffnung. Durch seine Unterhaltung mit dem Lampenträger bekommt der junge Mann die Gelegenheit, über seine Lebenseinstellung nachzudenken. Auch hat vielleicht der ältere Mann an die Worte des Jungen gedacht: "Das Leben ist mehr. . ."

Man findet in diesem Stück schöne Beispiele von Alliteration: "Und das Helle am Himmel in dieser Nacht, das Helle war Hamburg." "Und die runden rotierenden Räder rollten ratternd unter rostrosten roten Waggons." Man hört auch Assonanz: "Und sie machten die Nacht zur Nacht."

$$
\text { "Vier Soldaten"59 }
$$

Wie "Die drei dunklen Könige" gehört "Vier Soldaten" auch zum Zyklus An diesem Dienstag in der Gruppe "Im Schnee, im sauberen Schnee," und entstand im Jahre 1946.

Die Geschichte spielt in einem Schlupfloch, wo vier Soldaten einen anscheinend unvermeidbaren Tod von den Kanonen draussen erwarten. Ein öllicht, eine "Funzel," brennt schwankend; sonst ist alles dunkel.

Einer von den Soldaten hat blaue Lippen; als er die Kanonen hört, versucht er, einen Witz zu machen: "Hier 
braucht im Frühling aber nicht gepflügt zu werden." Ein anderer scheint, keine Angst $z$ u haben, aber er spricht von dem Tod: ". . wie findet ihr Radieschen? Die ganze Ewigkeit Radieschen?" Der dritte, "in der Ecke," spricht ernst: "Davon merkst du dann doch nichts mehr."

Der vierte Soldat sagt nichts. Er hält sich an seinem Gewehr fest, obwohI er dieses Gewehr hasst. Der vierte Soldat ist klein, und er hat einen Bart. Er leidet an "Hunger und Heimweh."

Der Soldat mit der Zigarette bittet um die ölfunzel, aber als der kleine ihm die Funzel reicht, fällt die Funzel hinunter.

$$
\text { "Und erlosch. Und erlosch." }
$$

Der kleine lacht laut: "Junge, habe ich einen Taterrich!"

Der in der Ecke denkt: "Keiner ist unter uns - . der nicht zittert."

Der mit der Zigarette (der "zuversichtliche") sagt: "Das kommt von der Kälte."

Als er die Kanonen hört, sagt der mit den blauen Lippen: "Die machen die ganzen Radieschen kaputt."

Und alle halten sich an den Gewehren fest, und Iachen in der Dunkelheit.

In "Vier Soldaten" ist es möglich, eine Umkehrung von "Die drei dunklen Könige" zu verstehen. In "Die drei dunklen Könige" ist eine Verwandlung von locus terribilis 
zum locus amoenus zu finden. 60 In "Vier Soldaten" findet man das Gegenteil. Die Lampe gewăhrt ein kleines Stückchen "locus amoenus;" wenigstens können die Soldaten ihre Gewehre sehen. Nach Jacobs ist die Lampe ein Zeichen der Menschlichkeit: "Das Menschliche ist in das Öllicht versetzt." 61

Aber die Lampe wird fallengelassen:

"Und erlosch. Und erlosch."

Und plötzlich ist es eine ganz furchterregende Welt: totale Dunkelheit: "locus terribilis."

Die Zentralität dieses Ereignisses wird durch die Wiederholung der Worte unterstrichen, und zwar ist es fast das einzige Ereignis der Geschichte. Koepke schreibt: ". . the plot of the stories seems intentionally non-dramatic."62 Kaszynski erklärt: "Die Handlung [in 'Vier Soldaten'] ist . . . bewegungslos . . .",63 und Jacobs sagt: "Es geschiet ja nichts."64 Aber es gibt doch dieses einzige Geschehen, was in diesem geschehensarmen Stück grosse Wichtigkeit hat.

Obwohl nach Jacobs alle vier Soldaten ohne Persönlichkeiten dargestellt sind, ${ }^{65}$ bekommen wir doch über den Vierten ziemlich viel persönliche Auskunft. Wir wissen, dass er klein ist, dass er sein Gewehr, mehr als irgendetwas in der Welt, hasst, dass die Lampe in seine Augen scheint, dass er Hunger und Heimweh hat.

Es ist vielleicht nicht unvorstellbar, in diesem 
kleinen Soldaten (auch der Soldat in "Hinter den Fenstern" war klein - Borchert aber nicht) ${ }^{66}$ Borchert selbst zu sehen; er hat früher gesagt: "Ich möchte Leuchtturm sein!" Leider ist er aber der, der die Lampe fallen lässt. Hier wird vielleicht Borcherts Bewusstsein seiner Schwäche noch einmal gespiegelt.

"Die Nachtigall singt"67

"Die Nachtigall singt" gehört zu der Gruppe, "Im Schnee, im sauberen Schnee," im Zyklus An diesem Dienstag. Diese Erzählung wurde im Jahre 1947 geschrieben.

Es ist Frühsommer; man riecht Flieder und hört die Nachtigall, und hört auch den Husten vom Herrn Hinsch, der wahrscheinlich einen "sommersüssen Tod" haben wird. Der Dichter spricht als Soldat und denkt an den Tod von einem Kameraden. Timm starb im Winter, "den einsam eisigen Wintertod." Timm hätte seinen Stahlhelm auf Posten tragen müssen, aber er hat anstatt "eine von seinen Weltreden" gehalten:

"Wir haben den Schnaps . . und die Stahlhelme - . und die chinesische Mauer und Lampen. . . [g]egen die Angst. . . [D]ie Dochte stecken wir aus Angst in òl und lassen sie brennen. . Aber helfen tut uns das nicht. . . [D]ann hilft uns kein Stahlhelm [auch keine Lampe] wenn die Angst uns erwischt."

Dann geht Timm fort, ohne seinen Stahlhelm. Als der 
Dichter später kommt, ihn abzulösen, findet er Timm tot im Schnee.

"Dann sagte ich zu dem toten Timm: Du hast recht, Timm, es hilft uns alles nicht." Er denkt jetzt daran, als er die Nachtigall hört. (Der Nachtigall ist der Tod von Timm und vom Herrn Hinsch egal.) Timm war gelb, wie Lehm. "(Ob die Nachtigall auch nur aus Lehm ist? So wie du, Timm?)"

In diesem kurzen Stück erscheint die Lampe als eine Schutzmassregel von der Angst. Aber Timm erklärt, das hilft nicht; auch der Stahlhelm hilft nicht, weil uns die Angst erwischt, wenn wir es nicht erwarten. Timm glaubte, man sollte versuchen, trotz seiner Angst mutig in die Dunkelheit zu gehen, und die Schutzmassregeln nicht zu verwenden, denn man würde sterben in der Stunde, in der man sterben sollte. Also trug Timm seinen Stahlhelm nicht, und er wurde vom Tod "erwischt."

Jetzt als der Schriftsteller an die heutigen Schutzmassregeln gegen die Angst (die Nachtigall, das Kreuz, den Flieder) denkt, erkennt er, dass diese Schutzmittel auch nicht helfen: trotz allen müssen alle Menschen sterben. Auch die Mittel selbst müssen sterben. Dieses Stück scheint also ziemlich verzweifelt $\mathrm{zu}$ enden, aber wir müssen uns an den Geist von Timms Leben erinnern, dass man das Leben wirklich leben muss, ohne Angst zu haben. Die Lampe usw. kann eine Quelle der 
Hoffnung sein, aber mann sollte davon nicht abhängen. Dieses Prinzip ist in Borcherts Werken klar zu sehen: auch wenn die Lampen erlöschen, geben die Menschen nicht auf.

$$
\text { "Im Mai, im Mai schrie der Kuckuck"68 }
$$

"Im Mai, im Mai schrie der Kuckuck" ist wahrscheinlich die letzte Geschichte von Borchert, ${ }^{69}$ und nach Klarmann eine der besten. ${ }^{70}$ Sie entstand im Jahr 1947 , und gehört zu der Gruppe "Und keiner weiss wohin," die im Band An diesem Dienstag nach Borcherts Tod verlegt wurde.

Dieses Stück ist länger als die anderen die in dieser Arbeit untersucht werden; es spielt nicht nur in einer Umgebung, sofondern in mehreren verschiedenen Rahmen. Es ist möglich, dieses Stück in bestimmte Einheiten zu gliedern, nämlich:

I. Vorspiel: vom Anfang bis S. 230: "Das ist das Buch . . ."

II. A ${ }^{1}$ : S. 230 "Aber die Abends . . " bis S. 231 ". . . in der Stadt geboren."

III. A $A^{2}$ : S. 231 "Nie wird die Welt. . . " bis S. 232 ". . das Versmass auf all . . ." IV. $\mathrm{B}^{1}: \mathrm{S} .232$ "Aber manchmal .. . ." bis S. 237 ". . die Augen verschwimmen . . " $\mathrm{B}^{2}$ : S. 237 "Es ist das übliche. . ." bis S. 241 ". . aber keiner erkennt uns. . ." V. Schluss: S. 243 "Der Mann, der gestern... ." bis Ende. 
Im I. Teil spricht der Dichter von den verschiedenen Stimmen im menschlichen Leben: im März hört man die Schiffe, im November die Lokomotive, im April die Katzen und die Mädchen. "All dies Weltgeschrei, . . an das erinnert man sich, das erträgt man. . . Aber . . im Mai der Kuckuck, wer unter uns erträgt. . . seinen.. . Schrei? . . [D]ann hilft kein Dampfer dir mehr und keine Lokomotive. . . Der Kuckuck lacht. . wohin? . . Und du stehst. . . allein, ohne wohin."

Im Schrei des Kuckucks hört man die tiefsten Sehnsüchte des Herzens. "[I]ch weiss, . . all dein Geschrei ist Geschrei nach der Mutter. . . ." Diese Gefühle lassen sich nicht in Worten ausdrücken: ". . das Letzte geben die Worte nicht her."

Der Dichter denkt an den Weltschmerz vom Krieg, den er im Kuckuckschrei hört, und er weiss, er hat für diesen Kriegsschmerz keine Worte. Es gibt ein Buch, worin notiert steht, was passiert ist. Aber die wirklichen Worte sind unsichtbar: " . . das Buch unserer . . Einsamkeit auf nachttoten Strassen."

Teile II und III stellen die Lebenseinstellung der "versorgten" Leute dem Lebensstil von denen gegenüber die, wenigstens im Herzen, noch auf den Strassen leben. "Aber -. die.. . in den . . Strassenbahnen, . . sie wollen ja irgendwohin. . . Sie möchten mit den Fahrschein spielen, wie Kinder, aber". . sie sind gute 
Schauspieler. . . und spielen Erwachsene. . . [D]ie müssen doch glücklich sein, denn sie sind ja geborgen."

Diese geborgenen Menschen sitzen unter Lampen, die als "nicht zu hell und. . . nicht zu triste" beschrieben werden. Die Lampen sind für diese "altgewordenen . . Kinder" die "mittelmässigen Gestirne ihrer Alltage." Diese "behördlich angeordneten Lampen" werden von dem "Vaterland" geschenkt, und damit sollen diese Leute zufrieden und froh sein: "Angst können sie nicht haben; Sie haben doch Licht." Sie sind nicht allein, weil sie einander haben; in der Strassenbahn hören sie auch den Kuckuck nicht.

Im Teil III stehen andere Leute allein auf den Strassen, "[o]hne Lampe, ohne Station, . . kuckucküberschrien, voll Angst." Die Strasse ist alles, was sie haben. Der Dichter identifiziert sich mit diesen Leuten: "Da stehen wir, dem Kuckuck ausgeliefert, . . . einsam - . muttersehnsüchtig, . - grosspurig, verloren. . . [U]ns fehlen die letzten Vokabeln. . auf all unsere Not."

Im IV. Teil, der sich weiter in drei Einheiten teilen lässt, ist der Kern dieses Stückes zu finden. Man könnte diesen Teil "Die Geschichte eines Soldaten" nennen. Im IV. Teil (der eine Beziehung mit dem II. zeigt) sieht der Mann auf der Strasse "(oh so selten)". . [e]in helles warmes . . Viereck. . in der fürchterlichen Schwärze der Nacht: Ein Fenster [mit einer Lampe]." 
In dieser Mainacht sieht der Mann eine Frau am Fenster, und er stellt sich vor, wie er mit ihr die Nacht verbringen könnte. Er hat Angst davor: er ist doch unerfahren. "Man muss das wie einen Schnaps runterkippen. . . ." "Alles andere ist rührseliges Geschwätz. - . Denn dies ist das Leben, für das es keine Vokabel gibt. . ."

Der Mann spricht mit der Frau. Zuerst ist er so nervös, dass er ihr nicht zuhört. Aber sie hat doch "ja" gesagt, und er geht in ihr Zimmer hinein. "Überm Tisch hängt die Lampe." Die Frau ist vielleicht vierundreissig; der Mann ungefähr zwanzig Jahre alt. Er sieht die Frau an, aber er sitzt vor Müdigkeit und Angst. Er winkt ihr, sie sollte die Lampe ausmachen. Aber auch im Dunkeln tut er nichts.

Die Frau bringt ihn zu dem Bett und "zieht ihn runter," aber er weiss einfach nicht, was er tun soll. "So ein Kind, denkt sie." Sie lässt ihn "verächtlich und mütterlich. . ." einschlafen.

Wie ein Kind schläft er die Nacht hindurch, mit seiner Hand zur Faust geballt. Beim Frühlicht sieht sie ihn an, und als sie den Mund ansieht,". . verschwimmen - . ihr die Augen."

Im $I^{2}$. Teil glaubt sie in ihrer Vorstellung, die Worte des Mundes zu hören:

Ich war nur müde gestern Abend; ich wusste genau, was 
zu tun, aber ich war einfach zu müde. "Aber. . ich bin schon lange ein Mann... Denn ich hab schon Wodka getrunken, . . ein Gewehr hab ich gehabt und Scheisse hab ich geschrien und geschossen hab ich und ganz allein auf Horchposten gestanden. . . "

Beim Abschied von den Eltern und den Bräuten hatten alle laut gesungen, denn". . . keiner riskierte im öllicht eine Träne."

Und kurz danach mussten sie kämpfen. "Und was dann kam, dafür gibts keine Vokabel, . . denn . . wer weiss einen Reim auf den Aufschrei eines achtzehnjährigen Mannes, - . mit seinen Gedärmen in den Händen zwischen den Linien - . , wer, denn, ach keiner!!!"

Auch jetzt nach dem Krieg finden sie keine Worte dafür. "Heute sagen wir einfach . . - das war der Krieg, - . denn uns fehlen die Vokabeln. . ." Der Krieg ist vorbei, und die Soldaten sind wieder zu Hause, aber sie sind ganz verändert; sie sind "altgewordene . . . Kinder." "Und keiner keiner kann uns noch erkennen, uns zwanzigjährige Greise. . ." Keiner, weder die Väter, noch die Mütter noch die Bräute, kann diesen Jungen jetzt verstehen. Teil IV ${ }^{3}$ bezieht sich auf Teil III, der auch von den Strassenleuten handelt. "Und jetzt sind wir unterwegs." Sie träumen von einem idealen Land. "Und wir sind unterwegs nach der ungebauten neuen Stadt, in der uns alle Fenster [wahrscheinlich mit ihren Lampen] gehören, und alle 
Fraven, und alles. . ." Und sie hoffen auf diese erleuchtete Stadt, ". . . in der alle hören und sehn und in der sich all verstehen... ." Aber nach dem Traum wissen sie, so eine Stadt gibt es nicht.

In diesem Moment beginnt der Junge aufzuwachen, und das Stück kommt zu Ende. Der Mann und die Frau wissen, sie leben in ganz verschiedenen Welten. "Und dafür gibt es keine Vokabel." Keiner von ihnen beiden spricht. "Der Schluss ist dann so wie alle wirklichen Schlüsse im Leben: banal, wortlos, uberwältigend."

Das Zeichen der Lampe scheint wichtig in den Teilen, die von Geborgenheit und Sicherheit handeln, nämlich in Teilen II und in $I V^{1}$ und $I V^{2}$.

Im Teil II werden die Lampen ziemlich gründlich beschrieben. Diese Lampen sind mittelmässig hell, und der Dichter betrachtet die Leute, die die Lampen erleuchten, als auch mittelmässig. Die Leute scheinen Erwachsene zu sein, aber sie sind doch kindisch, "albern(en), mürrisch(en)." Sie stehen im Gegensatz zu denen, die nach dem Krieg in den Strassen des Lebens bleiben (Teil IV ${ }^{2}$ ); diese scheinen jung $\mathrm{zu}$ sein, aber sie sind im Herzen alt geworden. Für sie liegen "zehntausend Jahre" zwischen dem Anfang und dem Ende des Krieges. "Dazwischen liegt unser Leben."

Diese mittelmässigen Lampen werden vom Vaterland gegeben; für die Durchschnittsmenschen reichen sie. Diese 
Menschen können sich auf die Regierung verlassen. Die Lampe in Teil IV gehørt zu der Mutter-Figur, nämlich der Frau am Fenster. Für den altgewordenen Jungen bedeutet die Lampe die Hoffnung auf Zuhause und Mutter und Geborgenheit. Durch das beleuchtete Fenster erlangt der Heimatlose eine Nacht unter den Geborgenen, weg von dem Kuckuckschrei, neben der Mutter. Denn wie Klarmann erklärt, der Soldat versucht erwachsen zu scheinen. "Awkward boyish playing at being a man. But. . a boy falls asleep on a mother's shoulder." 71

\section{Draussen vor der Tür ${ }^{72}$}

Borcherts bekanntestes Werk, und sein einziges Drama, ist Draussen vor der Tür. Es wurde im Januar 1947 oder Ende 1946 geschrieben, und am 13. Februar 1947 als Hörspiel gespielt. Am 21. November 1947, einen Tag nach Borcherts Tod, wurde es zum ersten Mal auf der Bühne aufgeführt, und in den folgenden Jahren erschien das Drama auf den Bühnen Deutschlands und in vielen anderen Ländern und Sprachen.

Das Stück handelt von der Heimkehr eines deutschen Soldaten (Beckmann) am Ende des Zweiten Weltkriegs. Nach einer kurzen Einführung entfaltet sich das Stück so: 
Vorspiel Beerdigungsunternehmer Gott

Traum (1): Elbe

1. Szene: der Andere das Mädchen

2. Szene: Mädchen Einbeiniger Anderer

3. Szene: Oberst

4. Szene: Kabarettdirektor der Andere

5. Szene: Fr. Kramer der Andere

Traum (2): Gott der Andere

Strassenfeger der Andere

Oberst der Andere

Kab.direktor der Andere

Beckmanns Frau der Andere

Mädchen, Einbeiniger der Andere

Epilog

Das "Vorspiel" Eindet am Ufer der Elbe, vor den St. Pauli Landungsbrücken in Hamburg statt. Es ist Abend und dunkel. Ein Beerdigungsunternehmer (der Tod) betrachtet einen jungen Mann (den heimkehrenden Soldaten Beckmann), der plötzlich ins Wasser springt. "Und . . keine Uhr bleibt stehen." Was er tut, ist der Welt und dem Tod egal. Dann sieht er aber einen alten Mann (Gott), der weint, "weil ich es nicht ändern kann. . . Es sind doch alles meine Kinder!"

"Der Traum" spielt in der Elbe, in die Beckmann 
gesprungen war. Dieser Fluss wird als eine Frau, und zwar als eine Mutter dargestellt. Beckmann darf nicht ". . unter [ihren] Rock kriechen," auch wenn seine Frau ihm untreu ist. Er muss weiterleben.

In der ersten Szene steht Beckmann am Strand bei

Blankenese in Hamburg. Er liegt am Ufer des Flusses. Die Figur "des Anderen" erscheint. (Nach Schulze"... symbolisiert [der Andere] einen anderen Teil von Beckmanns Ich, gleichsam seinen Lebensdrang."73 Nelson stimmt zu: "Der Andere [is] the externalization of Borchert's life instinct."74) Der Andere erklärt: "Ich bin der Optimist, der an den Bösen das Gute sieht und die Lampen in der finstersten Finsternis."

Beckmann erzählt dem Anderen seine Lage: er ist erst gestern aus der Kriegsgefangenschaft wieder nach Hause gekommen. Seine Frau ist ihm untreu, sein einjähriges Kind, das er nie gesehen hatte, liegt tot unter dem "Schuttacker," der von Hamburg übrig bleibt.

Ihr Gespräch wird unterbrochen von der Ankunft eines Mädchens, das Beckmann einlädt, er soll mit ihr nach Hause gehen und warme Kleider anziehen. Als sie den Strand verlassen, merkt der Andere, dass das Mädchen "im Dunkeln" [wie eine Lampe] zu Beckmann gekommen ist, und dass er jetzt ganz verändert ist. (Und zwar sagt Beckmann zu ihr gegen Ende des Stücks: "Du bist die Lampe, die für mich brennt.") 
Die zweite Szene fängt im Zimmer des Mädchens an. Sie will ihn unter der Lampe sehen. Sie nimmt seine Gasmaskenbrille $a b$ und sagt, er sähe besser aus, aber Beckmann klagt, er könne nichts richtig sehen. Das Mädchen versucht, ihn näher zu sich zu bringen, aber plötzlich sieht er hinter ihr die Gestalt ihres Mannes, der gerade aus dem Krieg gekommen ist. Er hat nur ein Bein.

Beckmann denkt daran, wie er seine Frau neben einem anderen Mann gefunden hat. "Da hab ich die Schlafzimmertür wieder zugemacht, nein, erst noch das Licht wieder ausgemacht."

Der Einbeinige geht aber nicht weg. Wie seine Frau will er Beckmann besser sehen: "Komm mit deinem Gesicht unter die Lampe. . . Beckmann!" Er erkennt Beckmann als den offizier, der für seine Verletzung verantwortlich ist. Beckmann läuft wieder auf die Strasse hinaus. Der Andere trifft ihn da und hindert ihn daran, wieder in die Elbe zu springen. Beckmann fühlt sich verantwortlich für die Kriegsschäden, aber er möchte diese Verantwortung wieder an den zurückgeben, der sie zuerst hatte: an den Oberst.

Die dritte Szene spielt in der Stube des Obersten. Der Oberst sitzt ("hinter den Fenstern") am Tisch mit seiner Familie. Durch "helle warme Fenster" hat Beckmann die Stube gefunden. Er versucht, seine Lage zu erklären; er beschuldigt den Oberst des Todes von Tausenden, und er 
will seine von dem Oberst bekommene Verantwortung zurückgeben.

Der Oberst glaubt, Beckmann mache einen Witz: er empfiehlt ihm, er solle auf die Bühne gehen, und bietet ihm saubere Kleidungsstūcke an. Beckmann wird sehr aufgeregt, und alle im Zimmer fangen an zu schreien.

In dem Aufruhr ist für diese versorgte Familie die Lampe vom grössten Belang. "Halt die Lampe fest!" Aber die Lampe wird umgeworfen. "Wo ist denn die Lampe? . . Gott sei Dank, dass wieder Licht ist."

Beckmann ist wieder auf der Strasse. Ermutigt von den Schnaps, den er vom Tisch des Obersten mitgebracht hat, entschliesst er sich, auf die Bühne zu gehen.

Also beginnt die vierte Szene im Zimmer des Direktors eines Kabaretts. Der Direktor spricht lange und kontradiktorisch, ${ }^{75}$ dass die Kunst die Jugend und die Wahrheit braucht. Aber als Beckmann sein Stück spielt, sagt der Direktor sofort, es ist zu traurig, und: "mit der Wahrheit hat die Kunst doch nichts zu tun!"

Noch einmal geht Beckmann an die Elbe, aber er wird von dem Anderen wieder zurück auf die Strasse gerufen, obwohl er sagt: "Nur die Strasse nach der Elbe runter, die ist hell." Der Andere erinnert ihn an das Haus seiner Eltern. Also geht Beckmann "[n]ach Hause, wo meine Mutter ist, meine Mutter - - - - "

In der fünften Szene findet sich Beckmann vor der Tür 
seiner Eltern. Statt seiner Mutter aber begegnet er der neuen Bewohnerin, Erau Kramer, eine "horrendous [mother-] substitute."76 Sie erzählt ihm ohne Mitleid die Geschichte des Selbstmords seiner Eltern.

Nach diesem Erlebnis versucht der Andere Beckmann noch einmal zu überzeugen, er solle auf der Strasse bleiben. Der Andere erklärt, viele Menschen trauern, wenn sie "unter ihren grünen Lampenschirmen" über den Tod anderer Menschen lesen. Beckmann erwidert, sie seufzen "unter ihren Lampen," aber "sie schlafen ruhig und tief, wenn sie noch ein Bett haben."

Der Andere erklärt: "Die Strasse wartet, Beckmann, komm!" In seiner Antwort sagt Beckmann: "Mensch, Mensch, was für eine Strasse bist du? Wo gehst du hin?" Der Andere versucht, ihm zu versichern: "[D]ie Strasse geht weiter und überall gibt es Lampen: . . Frauen, Fenster, Laternen. . . ." "Deine Strasse wartet. Und hin und wieder kommen Laternen. Bist du so feige, dass du Angst hast vor der Finsternis zwischen zwei Laternen? Willst du nur Laternen haben? Komm, Beckmann, weiter, bis zur nächsten Latèrne."

Aber trotz dieser Worte wird Beckmann sehr müde, und bald schläft er ein. Er stellt sich vor, dass er stirbt. In seinem Traum begegnet er noch einmal Gott, dem Tod (diesmal als Strassenfeger: "Heute liegen sie [die Toten] auf der Strasse...."), dem Oberst, dem Direktor des 
Kabaretts, Frau Kramer, seiner eigenen Frau und dem Mädchen und dem Einbeinigen.

Er verspottet Gott, und bittet den Tod darum, ihn nicht zu vergessen. Der Tod antwortet, er vergässe keine. Beckmann klagt den Obersten, den Direktor des Kabaretts und Frau Kramer an, sie seien für seinen Tod verantwortlich. Aber sie akzeptieren die Verantwortung nicht. Nach jeder Begegnung ermutigt ihn der Andere. Aber nachdem seine Frau an ihm vorbeigeht, ohne ihn zu erkennen, sagt er dem Anderen: "Alle Türen links und rechts der Strasse sind zu. Alle Laternen sind ausgegangen, alle. . . [H] thet du noch eine Laterne für mich in der Finsternis?"

Der Andere hat noch eine Lampe: das Mädchen kommt. Sie sagt $z u$ ihm, dass sie ihn liebt. Jetzt will er leben: "Du bist die Lampe, die für mich brennt. Für mich ganz allein. - . Und wir wollen ganz dicht nebeneinander gehen auf der dunklen Strasse." Und das Mädchen antwortet: "Ja, ich brenne für dich ganz allein auf der dunklen Strasse." Plötzlich aber sagt das Mädchen, sie darf nicht bleiben, und der Einbeinige nähert sich. Beckmann schreit, "Wo willst du denn hin?'. . Es ist ja auf einmal alles so dunkel! Lampe, kleine Lampe! Leuchte!" Aber statt des Mädchens hört er den Einbeinigen, der sich in der vorigen Nacht ertränkt hat, weil er Beckmann mit seiner Frau gefunden hat. Er zwingt Beckmann dazu, dass er verspricht, die Verantwortung für seinen Tod nicht zu vergessen. 
Dann wacht Beckmann auf, und er weiss, er lebt noch. Er fasst seine Lage zusammen und will wissen, warum er nicht sterben darf. Und wenn er nicht sterben darf, "wohin soll ich den? . . Wohin sollen wir denn auf dieser Welt!" Er ruft nach dem Anderen und nach Gott: "Gebt doch Antwort! Warum schweigt ihr denn? . . Gibt denn keiner, keiner Antwort?"

In Draussen vor der Tür findet man das Zeichen der Lampe besonders in Szenen eins, zwei und drei, und auch gegen Ende der zweiten Traumsequenz. "Der Andere" sieht die Lampe (also die Hoffnung) und hilft Beckmann, die Lampen zu finden. Also stellt der Andere eigentlich selbst eine "Lampe" für Beckmann dar. Nelson sagt sogar, der Andere erscheint nur, wenn Beckmann sich besonders verzweifelt fühlt. 77

Das Mädchen ist auch als "eine Lampe" zu verstehen: sie kommt zu ihm "im Dunkeln vorbei" und erleuchtet eine kurze Weile sein Leben. Gegen Ende des Stücks, wenn sie wieder zu ihm kommt, nennt er sie "die Lampe, die für mich brennt." Und sie stimmt zu. Sie darf aber nicht bleiben, weil ihr (im Traum) gestorbener Mann wieder zurück kommt, und Beckmanns "kleine Hoffnung (sinkt) wieder in sich zus ammen. 178

Wenn das Mädchen "eine Lampe" darstellt, hat sie auch zu Hause eine wirkliche Lampe. Wie in "Im Mai, im Mai schrie der Kuckuck," hofft der Strassenfahrer Beckmann 
darauf, dass er unter der Lampe ein Mass Geborgenheit finden kann. Es ist ihm aber nicht gelungen, weil der seit drei Jahren vermisste Mann des Mädchens in diesem Moment wieder nach Hause kommt, und im Schein der Lampe erkennt er Beckmann als denjenigen, der ihm befohlen hat, an seinem Posten zu bleiben; deswegen hat der Mann sein Bein verloren. Also muss Beckmann das haus und die Lampe(n) verlassen. Früher musste er sein eigenes Haus verlassen, als er seine Frau bei einem anderen Mann gefunden hat; zuerst hat er aber die Lampe (seiner Hoffnung) ausgelöscht. In diesem Stück, wie in "Im Mai, im Mai," gibt es Lampen für die "Ausgelieferten" und für die "Versorgten." In der Lampe, die er durch das "helle Fenster" des Obersten sieht, erwartet Beckmann, eine Lösung seines Problems zu finden, nämlich, dass der Oberst die Verantwortung für die Kriegsschäden zurücknehmen wird. Aber diese Lampe erweist sich als eine Lampe nur für die "Versorgten," und zwar, wenn später alle wegen Beckmanns Benehmen aufgeregt werden, wird für die Familie des Obersten die Lampe und das Licht eine Sache von höchster Wichtigkeit. "Gott sei Dank, dass wieder Licht ist." Wie die Strassenbahnleute in "Im Mai" sind diese Familienmitglieder als "altgewordene alberne - . Kinder" zu verstehen. "Angst können sie nicht haben: [denn] sie haben doch Licht."

Andere Lampen für die Versorgten erscheinen in der fünten Szene. Nach seiner Begegnung mit Frau Kramer ist 
Beckmann überzeugt, dass niemand in der Welt über den Tod von ein par Leuten wie seine Eltern trauert. Der Andere erklärt, doch, Leute lesen davon unter den Lampen und trauern. Aber Beckmann meint, sie trauern nicht lange; sie haben Betten, und trotz ihrer Trauer schlafen sie gut.

Wie auch in "Im Mai, im Mai" hängt die Lampe etwa mit dem Konzept der Mutter zusammen. Wie die Frau in "Im Mai" sieht das ("mother-surrogate") 79 Mädchen Beckmann "unter der Lampe." Und als Beckmann in der vierten Szene, nach seiner Begegnung mit dem Direktor des Kabaretts wieder zurück in die Elbe (auch eine Mutter-Figur) gehen wollte, erklärt er: "Nur die Strasse nach der Elbe . . . ist hell."

Nachdem Beckmanns Frau in der zweiten Traumsequen $z$ an ihm vorbei geht, scheint er wieder niedergeschlagen zu sein, und er drückt es so aus: "Alle Laternen sind ausgegangen, alle." Aber das Mädchen kommt wieder zu ihm zurück, und er bittet sie darum, als sein Lebenslicht zu bleiben: "Lampe, kleine Lampe! Leuchte!" Sie muss aber weggehen, weil ihr Mann kommt.

Nach diesem Vorfall scheint Beckmann ganz verzweifelt zu sein. Er braucht den Anderen, der aber diesmal nicht erscheint. Wenn, wie die meisten Kritiker meinen, der Andere als ein Teil oder eine Seite von Beckmann zu verstehen ist, muss er aber noch da sein, und zwar hat ex Beckmann versichert: "Du wirst mich nicht los."

Später hat der Andere erklârt: "Deine Strasse wartet. 
Und hin und wieder kommen Laternen. Bist du so feige, dass du Angst hast vor der Finsternis zwischen zwei Laternen? Willst du nur Laternen haben? Komm, Beckmann, weiter, bis zur nächsten Laterne."

Also sind die Laternen und auch die Lampen in Draussen vor der Tür wichtige Zeichen der Hoffnung. Für Beckmann ist die Hauptfrage die des Daseins. Nach Weimar ist Beckmanns Zweck "authentic existence." 80 Und Nelson versteht die Frage einfach als "to live or not to live."81 Für Schulze" . . stehen Beckmanns Lebenswillen seine Schuld- und Gewissenskomplexe. . . im Wege." 82

Beckmann "needs reasons not to commit suicide, not to give up." 83 Diese Gründe, die Laterne der Lebenshoffnung, werden einer nach dem anderen weggenommen. Am Ende des Stücks steht Beckmann allein auf der Strasse. Wie Marianne Schmidt bemerkt, ist er am Ende genau wo er am Anfang war, " - nur um einige Hoffnungen ärmer." 84

Schliesslich wichtig ist diese Beobachtung: in einigen von Borcherts Stücken scheint "die Strasse" wie "das Schlimmste." Der junge Mann in "Stimmen sind da" verlässt die Sicherheit der Strassenbahn und verschwindet aus unserer Sicht in die nebeligen Strassen. In "Hinter den Fenstern" steht der Soldat draussen in den Strassen, weil er zu feige, zu scheu ist, die notwendingen Schritte zu tun, um einen Platz "hinter den Fenstern" zu verdienen. Und der junge Mann in "Im Mai, im Mai" muss immer 
"unterwegs" durch die Strassen sein, weil sein vom Krieg verletztes Herz kein "Zuhause" finden kann.

Aber Beckmann steht auf der Strasse. Und für Beckmann ist das nicht das Schlimmste, denn die Strasse ist doch nicht die Elbe. Er hat mehrmals versucht, sich zu ertränken, aber der Andere hat ihm immer daran gehindert. Jetzt ist der Andere nicht zu sehen. Beckmann steht allein, am Nullpunkt seines Lebens; er könnte "zur Elbe" gehen, aber er bleibt auf der Strasse; er wählt das Leben. Und in diesem Zustand ist ein ". . message. . . of guarded hope" zu finden. 85 Weimar meint, es bleibt am Ende "no faith, no love, only hope."86 Carl-Heinz Corswandt sagte, Borchert wollte Beckmann keine leidende Figur, sondern einen Kämpfer darstellen. ${ }^{87}$ Zwar schrieb Borchert selbst in einem Brief im Februar 1947: "Geistig, seelisch, beruflich: Beckmann geht am Ende nicht in die Elbe. Er schreit nach Antwort! Er schreit nach Gott! Er fragt nach der Liebe! . . Und er bekommt keine Antwort. Es gibt keine. Das Leben selbst ist die Antwort." 88

Der Andere, ein Teil von Beckmann, der immer "eine Lampe" für Beckmann war, ist nicht zu sehen. Er muss aber da sein, also ist anzunehmen, er ist in Beckmann wieder verkörpert. Also wird "der Leuchtturm" mit dem "Schiff in Not" vereinigt, wie Borchert sich in seinem Gedicht gesehen hat: "Ich möchte Leuchtturm sein. . und ich bin doch selbst ein Schiff in Not!" Beckmann muss für sich selbst 
"Leuchtturm" sein, muss Lampen und Laternen selbst finden. "[D]ie Strasse geht weiter und überall gibt es Lampen:

- . Frauen, Fenster, Laternen. . ." 


\section{KAPITEL IV}

\section{SCHLUSSEOLGERUNGEN}

Was alle fühlen, muss der Eine sagen

für alle jubeln

und für alle klagen. ${ }^{1}$

In seinen Gedichten hat Borchert den Wunsch geåussert: "Ich mōchte Leuchtturm sein!" In seiner Prosa hat er diesen Wünsch erfüllt; gleichzeitig zeigte er sich als "ein Schiff in Not." Weimar sagt: "He was... a lantern in the night, no matter how feeble and flickering, a fullfilment of his own wish. . . ."2 Er wollte Hoffnung darstellen: "Despite the subject matter of Borchert's works, . . he intended his writing to be an affirmation of life." 3 Und in dieser "affirmation of life" hat er konsequent die Zeichen von Lampen und Laternen verwendet. Wenn man die Bedeutung von Lampen und Laternen in den Werken dieser Arbeit zusammenbringt merkt man, dass die "Lampen" gewöhnlich eine andere Rolle als die "Laternen" spielen. Die Lampen gehören oft (aber nicht immer) den "Versorgten," die entweder ein eigenes "Zuhause" oder "wohin" haben, wie in "Hinter den Fenstern," "Stimmen sind 
da," "Im Mai, im Mai" und Draussen vor der Tür. Die Lampen bedeuten die Sicherheit, die Liebe und das Wohlhaben. In "Die Nachtigall singt" gibt es keine wirkliche Lampe, aber wir erfahren, die Lampen sollen Angst hindern. In den Strassenbahnen bedeutet sie eine Art von "wohin:" diese Leute scheinen, ihren (Lebens-) Zweck zu kennen, obwohl die Strassenbahn nur eine zeitweise Zuflucht anbietet.

Andererseits kann man nicht behaupten, dass die Familie in "Die drei dunklen Könige" "Versorgte" sind, obwohl sie ein "Zuhause" haben. Ihre "Lampe" ist auch nur das Ofenlicht. Aber im Vergleich mit den drei

Heimkehrenden sind sie gut versorgt. Die Lage der "Vier Soldaten" ist fast eine Parodie von "Zuhause," und das Schlupfloch mit der Lampe stellt eine groteske Art von "Geborgenheit" dar. In "Die Stadt" gehört der ältere Mann auch zu denen, die ein "Zuhause" haben, also er hat eine "Lampe."

Die Lampen liefern die Möglichkeit für Kommunikation und ein Mass der Gemeinschaft, z.B. in "Stimmen sind da," "Die drei dunklen Könige" und "Die Stadt." In "Die drei" und "Stimmen sind da" ist die Zigarette, die Kommunikation erleichtert, als eine Art Lampe zu verstehen. In "Vier Soldaten" scheint die Lampe eine Hoffnung auf das Überleben selbst zu bedeuten.

Gewohnlich gibt es in diesen Werken einen "inneren Raum," der von einer Lampe erleuchtet ist. In "Gespräch" 
hat dieser innere Raum keine Lampe, und hier ist der erste Mann, der "tief im Zimmer" sitzt eine "Lampe" für den zweiten.

Die Figuren, die "draussen" stehen, haben keine Lampen; für diese Einzelgänger gibt es die Laternen. Diese "Aussenstehenden" hoffen immer auf die Lampen, obwohl sie wegen ihrer vorhergehenden Erlebnisse oft ausgeschlossen sind.

Fast jedes Stück ist von dem Gesichtspunkt dieser "Aussenstehenden" geschrieben. In "Hinter den Fenstern" bieten die Laternen $f u ̈ r$ den jungen Soldaten die Hoffnung auf Freundschaft und Liebe an. Der junge Mann in "Gespräch" steht am Fenster, also "draussen," und für ihn stehen die (Lampe-) Laternen im Hof und in den Strassen als Symbole der unzureichenden Führung der Regierung. (Die Strassenbahnlampen in "Stimmen sind da" und in "Im Mai" können auch so interpretiert werden.) Für den jungen Mann in "Die Stadt" ist das Leben noch ein Abenteuer; er hat keine Laterne, aber er geht mit voller Hoffnung zu den Laternen der Stadt. (Hamburg war doch für Borchert immer "Zuhause.")

Die "Aussenstehenden" und die "Versorgten" treffen sich oft: in "Gespräch," "Stimmen sind da," "Die drei," "Die Stadt" und in "Im Mai, im Mai." Gewöhnlich bekommen die Aussenstehenden einen Teil von dem Glühen der Lampe, aber in Draussen vor der Tür ist jede Begegnung mit den 
Versorgten eine Enttäuschung.

In "In Mai" wird die Laterne nicht erwähnt; wir lesen nur von "der fürchterlichen Schwärze der Nacht." 4 In Draussen vor der Tür spricht der Andere von Beckmanns Strasse, der Strasse seines Lebens, und von den Laternen, die da zu finden sind. Es ist klar, dass diese Laternen nicht in einem buchstäblichen sinn gemeint sind. Was bedeuten also diese Laternen?

Sie bedeuten, dass man weiter leben soll: sie sind eine Hoffnung auf Liebe, auf Geborgenheit, aber vor allem "[Borcherts] . . trotzige Hoffnung auf eine Veränderung der Menschen nach dem Krieg,"5 "seine.. Hoffnung auf das Gelingen eines neuen Anfangs, "6 eine bessere Zukunft. Borcherts Hoffnung spiegelt die des Mannes in "Gespräch:" ". . [M]orgen passiert es vielleicht, morgen kann es schon geschehen. . . Einmal kommt vielleicht eine Antwort. Und die, die soll ich versäumen? Nein, du, nein, nie!"7 Sein Ziel ist, durch die Nacht zu leben, die "European night," in der er schrieb.8

Borchert wollte vor allem die Wahrheit erklären. ${ }^{9}$ In seine "Nachwort" zu Draussen vor der Tür schrieb Heinrich Böll: "Die Wahrheit des Dichters, Borcherts Wahrheit ist, dass beide Schlachten, die gewonnene und die verlorene, Gemetzel waren. . . "10 Koepke erklärt: "Borchert... did not want to generate pity for the poor POW, . . but he was warning that the direction of society should not be 
entrusted to the old establishment."11 Borchert selbst schrieb: "Der vorlezte Akt der menschlichen Tragödie ist zu Ende gegangen. Ob der letzte Akt die Vernichtung oder Auferstehung bringen wird, das ist die Frage, die wie ein riesiger Schatten über uns allen liegt."12

Trotz dieses Schattens hoffte Borchert noch, und seine Charaktere hoffen auch. "[W]e find in Borchert's work a determination to carry on in spite of everything. . . "13 Die "Vier Soldaten" lachen in der Dunkelheit. der junge Mann in "Im Mai, im Mai" ist wieder "unterwegs." Und der junge Soldat in Draussen vor der Tür geht nicht in die Elbe, sondern er steht "kämpferisch" auf der Strasse des Lebens.

Schliesslich kann man sagen, Borchert wollte betonen, die Menschheit hat die Verantwortung (kein "leeres Wort") 14 für ihre Hoffnung auf die Zukunft:

Es soll heissen, sie haben uns zwar blind in diesen Krieg gehen lassen, aber nun wissen wir Sehen-gewordenen, dass nur noch eine Ankunft zu neuen Ufern uns retten kann, mutiger gesagt: Diese Hoffnung gehört uns allein! 15 
ANMERKUNGEN

KAPITEL I

${ }^{1}$ Wulf Koepke, "In Sachen Wolfgang Borchert," Studies in German: In Memory of Andrew Lewis, ed. Robert L. Kahn. Rice University Studies 55, iii (1969): 88 .

2 Koepke, "German Writers after 1945: Wolfgang Borchert," German Studies Review 2 (1979): 54.

3 Peter Rühmkorf, Wolfgang Borchert (Hamburg: Rowohlt, 1961) 29.

${ }^{4}$ Rühmkorf 29.

${ }^{5}$ Rühmkorf 48 .

${ }^{6}$ Rühmkorf 102.

7 Rühmkorf 66 .

${ }^{8}$ Rühmkorf 77.

${ }^{9}$ Rühmkorf 79 .

$10_{\text {Rühmkorf } 86 .}$

$11_{\text {Rühmkorf }} 92$.

12 Rühmkorf 115.

13 Rühmkorf 107.

${ }^{14}$ Rühmkorf 117 .

15 Joseph Mileck, "Wolfgang Borchert: "Draussen vor der Tür": A Young Poet's Struggle with Guilt and Despair," Monatshefte Li (1959): 329 . 
16 Rühmkorf 132 .

17 Rühmkorf 146 .

18 Rühmkorf 133.

19 Rühmkorf 148 .

${ }^{20}$ Irmgard Schindler, Unterhaltung mit der Verfasserin, 28. Marz 1985.

${ }^{21}$ Professor Dr. Alfred Läpple, Brief an die Verfasserin, 3. Mai 1985 .

${ }^{22}$ Schwester Mina Herbel, Brief an die Studentinnen einer Mitschwester, 23. Februar 1971.

\section{KAPITEL II}

$1_{\text {Rühmkorf } 30 .}$

2 Wolfgang Borchert, Das Gesamtwerk (Hamburg: Rowohlt,

1949) 7. Fleete sind Kanale in Hamburg.

3 Gesamtwerk 274 .

${ }^{4}$ Koepke, "In Sachen" 78.

5 Mileck 329.

6 Kurt J. Fickert, Signs and Portents: Myth in the

Work of Wolfgang Borchert (Fredricton, N.B.: York, 1980) 41.

7 Gesamtwerk 12 .

${ }^{8}$ R.S. Furness, Expressionism. The Critical Idiom 29,

John D. Jump, gen. ed. (London: Methuen, 1973) 16.

${ }^{9}$ Furness 42.

10 Rühmkorf 36. Das ganze Gedicht ist in der Staatsund Universitätsbibliothek Hamburg. 
${ }^{11}$ Gesamtwerk 171.

12 Furness 4.

13 Gesamtwerk 277 .

${ }^{14}$ Gesamtwerk 300 .

15 Gesamtwerk 144 .

${ }^{16}$ Edward Allen McCormick, "Thematic and Lexical

Unity in Wolfgang Borchert," Kentucky Foreign Language Quarterly viii (1960): 70 .

17 Wilhelm Jacobs, Moderne Dichtung: Zugang und Deutung (Gütersloh: Signum, 1962) 164.

18 Wolfgang Borchert, "Die traurigen Geranien" und andere Geschichten aus dem Nachlass (1962; Hamburg: Rowohlt, 1983) 96-99.

${ }^{19}$ Adolph D. Klarmann, "Wolfgang Borchert: The Lost Voice of a New Germany," Germanic Review xxvii (1952): 111. ${ }^{20}$ Karl S. Weimar, "No Entry, No Exit: A Study of Borchert with some Notes on Sartre," Modern Language Quarterly xvii (1955): 165 .

${ }^{21}$ Julius Bab, über den Tag Hinaus (Heidelberg: Lambert Schneider, 1960) 235.

22 Koepke, "In Sachen" 87.

23Marianne Bonwit, "Wolfgang Borchert, 1921-1947," Books Abroad xxv (1951): 15 .

24 McCormick 69. 
KAPITEL III

${ }^{1}$ Gesamtwerk 5 .

${ }^{2}$ Carl-Heinz Corswandt, Unterhaltung mit der

Verfasserin, 28. März 1985.

3 Rühmkorf 147 .

${ }^{4}$ Schindler, Unterhaltung.

5 Koepke, "German Writers" 60.

6ilhelm Grenzmann, Dichtung und Glaube (Bonn:

Athenäum, 1950) 308 .

${ }^{7}$ Stefan H. Kaszynski, Typologie und Deutung der

Kurzgeschichte von Wolfgang Borchert (Poznam: Universität im. Adam Miekiewieza, 1970) 120.

${ }^{8}$ Mileck 329.

${ }^{9}$ Rühmkorf 103.

${ }^{10}$ Franz Langhammer, Unterhaltung mit der Verfasserin, 30. Juni 1985 .

${ }^{11}$ Corswandt, Unterhaltung.

$12 \mathrm{Bab} 236$.

13 Fickert, "Signs and Portents" 41.

${ }^{14}$ Rühmkorf 102, 104.

15 Rühmkorf 97 .

16 Rühmkorf 154.

17 Gesamtwerk 7 .

${ }^{18}$ Gesamtwerk 274 .

${ }^{19}$ Gesamtwerk 276.

20 Fickert, "Signs and Portents" 43. 
$21_{\text {Gesamtwerk } 277 .}$

22 Klarmann 111.

23 McCormick 69.

${ }^{24}$ Corswandt, Unterhaltung.

25 Koepke, "German Writers" 55.

26 Mileck 328.

27 Mileck 328 .

28 McCormick 68 .

29 Klarmann 112.

30 Koepke, "In Sachen" 78.

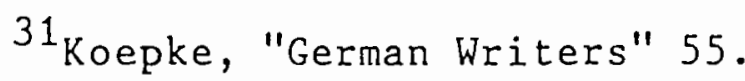

32 Fickert, "Wolfgang Borchert's 'Billbrook' as a

Search for the Self," Books Abroad XL (1966): 31.

$33_{\text {McCormick } 74 .}$.

${ }^{34}$ Gesamtwerk 48-55.

35 Klarmann 115 .

36 McCormick 69.

37"Die traurigen Geranien" 96-99.

$38_{\text {Rühmkorf } 132 .}$

39 "Geranien," Vorwort 2.

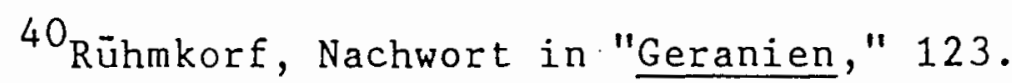

${ }^{41}$ Gesamtwerk 44-48.

${ }^{42}$ Károly Csuri in Arpád Bernáth, Károly Csuri und Zoltan Kanyó, Textheorie und Interpretation: Untersuchung zu Gryphius, Borchert und Böll (Kronberg: Scriptor, 1975) 
${ }^{43}$ Csuri 205.

${ }^{44}$ Alfred Schmidt, Wolfgang Borchert: Sprachgestaltung in seinem Werk (Bonn: Bouvier, 1975) 183 .

45 Csuri 179 .

${ }^{46}$ Csuri 200 .

47 Csuri 202 .

${ }^{48}$ Gesamtwerk 185-187.

${ }^{49}$ Werner Zimmermann, Deutsche Prosadichtungen der

Gegenwart, Teil II (Düsseldorf: Schwann, 6. Auflage 1960) 124.

50 Kaszynski 143 .

51 Zimmerman 125 .

${ }^{52}$ Schindler, Unterhaltung.

${ }^{53}$ Robert Pichl, "Topische Elemente in den Kurzgeschichten Wolfgang Borcherts," Sprachkunst 6 (1974): 369.

54 Kaszynski 82 .

55 Zimmermann 122 .

56 Zimmermann 124 .

57 Karl Migner, "Wolfgang Borchert," Welt und Wort 16 (1961): 139 .

58 Gesamtwerk 69-71.

${ }^{59}$ Gesamtwerk $171-172$.

60 Pichl 179.

61 Jacobs 161 .

62 Koepke, "German Writers" 55.

63 Kaszynski 49 . 
64 Jacobs 162 .

65 Jacobs 163.

${ }^{66}$ Corswandt, Unterhaltung.

67 Gesamtwerk 183-185.

${ }^{68}$ Gesamtwerk 26-243.

${ }^{69}$ Rühmkorf 133.

$70_{\mathrm{K} l a r m a n n} 119$.

71 Klarmann 120.

72 Gesamtwerk 99-165.

73 Wolfgang Schulze, "Draussen vor der Tür," Wirkendes Wort $13(1963): 119$.

${ }^{74}$ Donald Nelson, "To Live or Not to Live: Notes on Archtypes and the Absurd in Borchert's Draussen vor der Tür, " German Quarterly 48 (1975): 346. 75 Nelson 350 .

$76 \mathrm{Nelson} 350$.

$77 \mathrm{Nels}$ on 347 .

${ }^{78}$ Paul Fechter, Vom Expressionismus zur Gegenwart aus den Nachlass herausgegeben. Das Europäische Drama: Geist und Kultur im Spiegel des Theaters 3 (Mannheim:

Bibliographisches Institut, 1958) 191.

${ }^{79} \mathrm{Nels}$ on 348 .

80 Weimar 153 .

81 Nelson 343 .

${ }^{82}$ Schulze 121

83 Koepke, "German Writers" 56. 
${ }^{84}$ Marianne Schmidt, "Probleme des Humanismus und der Prospektus im Schaffen Wolfgang Borcherts," diss., Greifswald 1966, 143.

85 Koepke, "German Writers" 61.

86 Weimar 165 .

87 Corswandt, Unterhaltung.

${ }^{88}$ Borchert, "Briefe aus dem Nachlass," Akzente 2 $(1955): 118$.

KAPITEL IV

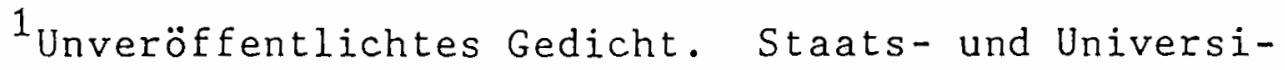
tätsbibliothek Hamburg. Dieses Gedicht wurde 1942 im Untersuchungsgefängnis Nürnberg geschrieben.

${ }^{2}$ Weimar 160 .

${ }^{3}$ Fichert 167 .

${ }^{4}$ Gesamtwerk 233 .

5 Marianne Schmidt, "Anklage und Hoffnung. Wolfgang Borchert 20. Mai 1921 - 20. November 1947," Neue deutsche Literatur (Ost-Berlin) 12 (1967): 95.

6igner 138 .

${ }^{7}$ Gesamtwerk 56 .

8 Bonwit 18 .

${ }^{9}$ Corswandt, Unterhaltung.

$10_{\text {Heinrich Böll, Nachwort, Draussen vor der Tür und }}$ andere ausgewählte Erzählungen, von Wolfgang Borchert (Hamburg: Rowohlt Taschenbuch, 1956) 119.

11 Koepke, "German Writers" 61. 
12 Helmut Gumtau, Wolfgang Borchert (Berlin:

Colloquium, Otto H. Hess, 1969) 66.

13 Koepke, "German Writers" 60.

${ }^{14}$ Gesamtwerk 126 .

15 Rühmkorf 159. 
Ahl, Herbert. Literarische Porträts. München: Langen-Mueller, 1962.

Anders, William. "Heimkehrsituationen nach zwei Weltkriegen." German Life and Letters vii (1954): $170-179$.

Bab, Julius. Über den Tag Hinaus. Heidelberg: Schneider, 1960.

Bernáth, Arpád, Károly Csuri und Zoltan Kanyó. Texttheorie und Interpretation: Untersuchung zu Gryphius, Borchert und Böll. Kronberg: Scriptor, 1975.

Bonwit, Marianne. "Wolfgang Borchert, 1921-1947." Books Abroad xxv (1951): 15-18.

BöIl, Heinrich. "Die Stimme Wolfgang Borcherts." Nachwort. Draussen vor der Tür und andere ausgewählte Erzählungen. Hamburg: Rowohlt Taschenbuch, $1956,118-120$.

Borchert, Wolfgang. "Briefe aus dem Nachlass." Akzente 2 (1955): 116-119.

-.. "Die traurigen Geranien" und andere Geschichten aus dem Nachlass. 1060. Reinbek by Hamburg: Rowohlt Taschenbuch, 1967.

---. Das Gesamtwerk. Hamburg: Rowohlt, 1949.

.... Unveröffentliche Gedichte. Staats- und Universitätsbibliothek Hamburg.

Bortonschlager, Wilhelm. Autoren aus Westdeutschland und aus Ostdeutschland. Teil 3 vom Theaterspiegel. München: Welsermühl, n.d.

Brunet, Georges. "La lange de Wolfgang Borchert: Realismé et Realité," in: Paul Vernais, ed. Le Reel dans la litterature et dans la langue. Paris: Klincksieck (1967): 245-47. 
Corswandt, Carl-Heinz. Unterhaltung mit der Verfasserin. 28. Marz 1985.

Daniel-Rops, Henry. Where Angels Pass. tr. Emma Crawford. London: Cassell, 1950.

Darboven, Anna-Maria. Wolfgang Borchert: Der Rufer in einer Zeit der Not. Hannover: Gödel, 1957.

Fechter, Paul. Vom Expressionismus zur Gegenwart aus dem Nachlass herausgegeben. Teil 3 von Das Europäische Drama: Geist und Kultur im Spiegel des Theaters. Mannheim: Bibliographisches Institut, 1958.

Fickert, Kurt J. "The Christ-Figure in Borchert's Draussen vor der Tür." Germanic Review 54 $(1978): 165-69$.

--. Signs and Portents: Myth in the Work of Wolfgang Borchert. Fredricton, N.B.: York, 1980.

-.. "Some Biblical Prototypes in Wolfgang Borchert's Stories." German Quarterly xxxviii (1965): $172-178$.

-.-. "Wolfgang Borchert's 'Billbrook' as a Search for the Self." Books Abroad XL (1966): 31-34.

Frerking, Johann. Augenblick des Theaters. Velber bei Hannover: Friedrich, 1963.

Eurness, R.S. Expressionism. Gen ed. John D. Jump, The Critical Idiom 29. London: Methuen, 1973.

Grenzmann, Wilhelm. Dichtung und Glaube. Bonn: Athenaeum, 1950 .

Gsteiger, Manfred. Literatur des Übergangs. Bern: Francke, 1963.

Gumtau, Helmut. Wolfgang Borchert. Köpfe des 20. Jahrhunderts 55. Berlin: Colloquium, Ot to Hess, 1969 .

Heller, Erich. The Disinherited Mind. Philadelphia: Dufour and Saifer, 1952.

Herbel, Sr. Mina. Brief an die Studentinnen einer Mitschwester. 23. Eebruar 1971. 
Herd, E.W. "Wolfgang Borchert." German Life and Letters $4(1950-51): 295-98$.

Höller, Walther. "Die kurze Forme der Prosa." Akzente 9 (1962): 226-45.

Jacobs, Wilhelm. Moderne Dichtung: Zugang und Deutung. Gutersioh: Signum, 1962.

Jung, Hermann. "Wolfgang Borchert zum Gedächtnis: Zum zehnjährigen Todestag am 20. November 1957.

Schweizer Monatshefte xxxvii (1957): 695-702.

Kaszynski, Stefan H. "Die Lyrik von Wolfgang Borchert." Studia Germanica Posnamiensia 1 (1971): 13-25.

--- Typologie und Deutung der Kurzgeschichten von Wolfgang Borchert. Poznam: U in Adam Miekiewieza, 1970 .

Kilchermann, Ruth. Die Kurzgeschichte: Form und Entwicklungen. Stuttgart: Kohlhammer, 1967.

Klarmann, Adolph D. "Wolfgang Borchert: The Lost Voice of a New Germany." Germanic Review xxvii (1952): 108-123.

Koepke, Wulf. "German Writers after 1945: Wolfgang Borchert." German Studies Review 2 (1979): 49-62.

-.-. "In Sachen kiolfgang Borchert." Ed. Robert L. Kohn. Studies in German: In Memory of Andrew Lewis. Houston: Rice University Studies 55, iii $(1969)$ : $69-71$.

Krell, Leo, u. Leonhard Fiedler. Deutsche Literaturgeschichte. Bamberg: Buchner, 15. Aufl. 1973.

Langhammer, Franz. Unterhaltung mit der Verfasserin. 30. Juni 1985.

Läpple, Prof. Dr. Alfred. Brief an die Verfasserin. 3. Mai 1985 .

Mandel, Siegfried. "The German Novel: In the Wake of Organized Madness." Ed. Siegfried Mandell. Contemporary European Novelists. Carbondale: Southern Illinois U P (1968): 69-125. 
--. Group 47: The Reflected Intellect. Carbondale: SIU P, 1973 .

McCormick, Edward Allen. "Thematic and Lexical Unity in Wolfgang Borchert." Kentucky Foreign Language Quarterly viii (1961): 66-74.

Melchinger, Siegfried. Theater der Gegenwart. Frankurt am Main: Fischer, 1956.

Meyer-Marwitz, Bernhard. Nachwort. Das Gesamtwerk, Wolfgang Borchert. 1947. Hamburg: Rowohlt, 1957: $325-349$.

Migner, Karl. "Wolfgang Borchert: 'Draussen vor der Tür': A Young Poet's Struggle with Guilt and Despair." Monatshefte Li (1959): 328-336.

-.. "Wolfgang Borchert's Bibliography." German Quarterly xxxiii (1960): 233-239.

Nelson, Donald F. "To Live or Not to Live: Notes on Archtypes and the Absurd in Borchert's Draussen vor der Tür." German Quarterly 48 (1975): 343-54.

Padgett, Jacqueline 0 . "The Poet in War: Walt Whitman and Wolfgang Borchert." Monatshefte 72 (1979): 149-61.

Peters, H.F. Rainer Maria Rilke: Masks and the Man. Seattle: U of Wash P, 1960.

Pichl, Robert. "Das Bild des Kindes in Wolfgang Borcherts Prosa." Jahrbuch für Internationale Germanistik 2, iii $(1975): 469-74$ [Ser. A.]

-.. "Topische Elemente in den Kurzgeschichten Wolfgang Borcherts." Sprachkunst 6(1974): 362-72.

Pinault, Michel. "Wolfgang Borchert et l'angoisse du temps present." Etudes Germaniques xi (1956): $36-44$.

Reid, J.H. "Draussen vor der Tur in Context." Modern Languages 61 (1979): 184-90.

Rilke, Rainer Maria. Das Stunden-Buch. 1905. Leipzig: Insel Taschenbuch, 1980.

Rühmkorf, Peter. Wolfgang Borchert. Reinbek bei Hamburg: Rowohlt Taschenbuch, 1961. 
Schindler, Irmgard. Unterhaltung mit der Verfasserin. 26., 28. März 1985.

Schlosser, Horst Dieter. Atlas zur deutschen Literatur. München: Deutscher Taschenbuch, 1983.

Schmidt, Adalbert. Literaturgeschichte: Wege und Wandlungen Moderner Dichtung. Stuttgart 1957. Salzburg: Bergland-Buch, 1959.

Schmidt, Alfred. Wolfgang Borchert: Sprachgestaltung in seinem Werk. Bonn: Bouvier, 1975.

Schmidt, Marianne. "Anklage und Hoffnung. Wolfgang Borchert 20. Mai 1921 - 20. November 1947." Neue deutsche Literatur (Ost-Berlin) 12 (1967): $85-9 \overline{7}$.

--. "Probleme der Humanismus und der Prospektus im Schaffen Wolfgang Borcherts." Diss. Phil. Greifswald, 1966 .

Schnurre, Wolfdietrich. "Kritik und Waffe" zur Problematik der Kurzgeschichte." Deutsche Rundschau 87 (1961): $61-66$.

Schulze, Wolfgang. "Draussen vor der Tür." Wirkendes Wort 13 (1963): 115-122.

Sorgel, Albert, u. Curt Hohoff. Vom Naturalismus bis zur Gegenwart. Teil 2 von Dichtung und Dichter der Zeit. Düsseldorf: Bagel, 1963.

Spaethling, Robert H. "Wolfgang Borchert's Quest for Human Freedom." German Life and Letters xiv (1961): 188-93.

Weber, Warner. Figuren und Fahrten. Zürich: Manesse, Conzett u. Huber, 1956.

Weimar, Karl S. "No Entry, No Exit: A Study of Borchert with some Notes on Sartre." Modern Language Quarterly xvii (1956): 153-63.

Willson, A. Leslie. "Beckmann, der Ertrinkende: zu Wolfgang Borcherts 'Draussen vor der Tür.' Akzente 19 (1972): 466-79. 
Wolff, Rudolf, herausgeber. Wolfgang Borchert: Werk und Wirkung. Bonn: Bouvier, 1984 .

Zimmerman, Werner. Deutsche Prosadichtungen der Gegenwart. Teil 2. Düsseldorf: Schwann, 1956. 
APPENDIX A

CHRONOLOGISCHE LISTE VON BORCHERTS PROSAWERKEN

1946 Die Hundeblume

Tui Hoo

Alle Milchleute heissen Hinsch

Hamburg

Nasenbein

Marguerite

Ein Sonntagmorgen

Eisenbahn, nachmittags und nachts

Generation ohne Abschied

Gespräch uber den Dächern

Von drüben nach drüben

Billbrook

Schischyphusch

Die Elbe

Jesus macht nicht mehr mit

Das Brot

Hinter den Fenstern ist Weihnachten

Gottes Auge

Stimmen sind da - in der Luft - in der Nacht

Spatër Nachmittag

Die drei dunklen Könige 
Preussens Gloria

Die Krähen fleigen abends nach Hause

Vorbei vorbei

Bleib doch, Giraffe

Die Stadt

Vier Soldaten

Die traurigen Geranien

Nachts schlafen die Ratten doch

Draussen vor der Tür

Die Kỉchenuhr

Die Kirschen

Das Holz für morgen

Der viele viele Schnee

Das Känguruh

Die Kegelbahn

An diesem Dienstag

Lesebuchgeschichten

Vielleicht hat sie ein rosa Hemd

Die lange lange Strasse lang

Die Nachtigall singt

Der Kaffee ist undefinierbar

Das ist unser Manifest

Die Katze war im Schnee erfroren

Er hatte auch viel Ärger mit den Kriegen

Mein bleicher Bruder

Unser kleiner Mozart 
(1947) Der Schriftsteller

Maria, alles Maria

Im Mai, im Mai schrie der Kuckuck

Dann gibt es nur eins

ohne Chronologie:

Der Rahmbonbon

Die Professoren wissen auch nix

Liebe blaue grave Nacht

Das Gewitter

Ching-Ling die Fliege

Unser Pusteblumendasein 
APPENDIX B

ABENTEUER IN HAMBURG:

AUF DER SUCHE NACH WOLFGANG BORCHERT

Wo muss man anfangen, wenn man die Vorgeschichte über eine bestimmte Person erfahren möchte? Betreffs des frühgestorbenen Schriftstellers Wolfgang Borchert gibt es nur eine Antwort: seine von ihm sehr geliebte Heimatstadt Hamburg. Anfang Marz $1985 \mathrm{kam}$ ich also in diese grosse Stadt, um alles Mögliche über Borchert zu lernen.

Zuerst ging alles langsam. Hamburg war im Mărz ziemlich dunkel, und mein Thema, Lampen und Laternen, passte gut $z u$ der Umgebung. Niemand in Hamburg schien viel über Borchert zu wissen! Ich rief den Biographer Peter Rühmkorf an, aber er sagte, er wäre sehr beschäftigt und hätte fast alles vergessen!

Ich besuchte die Bibliothek. Dort kann man ein Buch sehr schnell mit dem Computer bestellen; leider kann man das Buch erst am năchsten Tag bekommen! (langsam ...) Nach ein paar solchen Besuchen gab ich entmutigt auf.

Zum Glück hatte ich von einer Bekannten in Bayern den Namen einer älteren Hamburgerin bekommen. Als ich diese wirklich "gnädige Frau" besuchte, bekam ich von ihr Zeitungsausschnitte über Borcherts Mutter, die kurz vorher 
gestorben war. In Zeitungsausschnitten fand ich eine Erwähnung von einem "Borchert-Zimmer" in der Bibliothek! Mit grosser Hoffnung lief ich wieder zur Bibliothek zurück. Der Mann am Auskunftsschalter war freundlich: "Sicher! Rufen Sie einfach diese Telefonnumer an!"

Ich ging nach Hause und rief an. Niemand meldete sich. Am nächsten Tag rief ich wieder an. Keine Antwort. ("Gibt keiner, keiner Antwort?") Nach einer Woche rief ich die Bibliothek an. "Ich bin wirklich enttãuscht," klagte ich; "ich bin drei Wochen in dieser Stadt, und ich kann niemanden finden, der irgendein Interesse für Wolfgang Borchert hat!" Natürlich wollte das niemand hören; die nette Dame, mit der ich sprach, sagte mir sofort, niemand im Borchert-Zimmer hatte sich gemeldet, weil das Zimmer "wegen Umbau geschlossen" war. Das Zimmer war im Moment einfach nicht da.

Aber sie gab mir die Telefonnummer von Frau Irmgard Schindler, eine gute Freundin von Borcherts Mutter, die mit der Führung des Borchert-Zimmers beauftragt war. Ich rief sie an, und am nächsten Tag traffen wir uns in der Bibliothek.

Durch Frau Schindler veränderte sich mein Glück. Zusammen untersuchten wir die Schränke, worin alles vom Borchert-Zimmer eingelagert war, und wir nahmen alles heraus, das ich für meine Arbeit brauchte. Mir wurde eine private Arbeitsstelle gegeben, und ein Beamter der Bibliothek wurde zu meiner Verfügung gestellt. Dieser 
rücksichtvolle Mann beschaffte mir sofort jedes Buch in der Bibliothek, das ich brauchte, ohne einen Tag darauf warten zu müssen! Auch erklärte er mir das System, z.B., dass man ein Buch, das Die kurze Form der Prosa heisst, unter ( $F$ )orm finden würde, während in den Vereinigten Staten dieser Titel unter (k)urze zu finden ist.

Also fand ich in der Staats- und Universitätsbibliothek Hamburg viel mehr als ich erwartet hatte. Aber vor allem half mir Frau Schindler selber. Von ihr bekam ich alle Auskunft über die drei "Wolfgang Borchert" Schulen, über das Geburtshaus und die Friedenseiche, und über die Stelle des Grabs auf dem Ohlsdorfer Friedhof. Sie teilte mir viele Einzelheiten über Borchert mit, und gab mir eine Kopie von Borcherts "Selbst-Porträt," das eine Frau, eine Gruppe von Stadtgebäuden und eine Strassenlaterne enthält!

Sie gab mir auch die Telefonnummer seines Vetters Carl-Heinz Corswandt, und am nächsten Tag rief ich Herrn Corswandt an. "Bitte, wann darf ich kommen?" "Sie dürfen sofort kommen! Erau Schindler hat mich schon angerufen!" Natürlich ging ich "sofort" hin, und lernte diesen lieben Menschen kennen. Wir unterhielten uns eine Stunde über verschiedene Aspekte von Borcherts Leben. Am Ende unseres Gesprächs liess ich eine Aufnahme mit diesem grossen Mann machen. Wolfgang Borchert war auch ungefähr so gross: fast zwei Meter!

Bevor ich Hamburg verliess, gelang es mir, das 
Geburtshaus, die Friedenseiche und die Borchert Schule zu besuchen (und später auch die Schule in Berlin), sowohI als auch das Grab; dort, im strömenden Regen, legte ich meinen kleinen gelben Blumenstrauss nieder: "winzige, unscheinbare Sonnen." (aus "Die Hundeblume"). 